\title{
NONEXPONENTIAL LEAVES AT FINITE LEVEL
}

\author{
BY \\ JOHN CANTWELL ${ }^{1}$ AND LAWRENCE CONLON ${ }^{2}$
}

\begin{abstract}
Previous examples of leaves with nonexponential and nonpolynomial growth (due to G. Hector) have occurred at infinite level. Here the same growth types are produced at finite level in open, saturated sets of leaves without holonomy. Such sets consist of leaves with only one or two locally dense ends, and it is shown that the exotic growth types only occur in the case of one locally dense end. Finally, $C^{1}$-foliations are produced with open, saturated sets as above in which the leaves have strictly fractional growth.
\end{abstract}

Introduction. In [He1] it was shown how to construct $C^{\infty}$-foliations in which a continuum of distinct, nonexponential growth types occurs simultaneously. In these constructions, the interesting growth types (nonpolynomial and nonexponential) occur for leaves at infinite level, a notion to be made precise in $\$ 1$. We will show (\$5) how to produce continuously many distinct nonpolynomial growth types at finite level, although not simultaneously in one foliation.

The theory of levels and of totally proper leaves [C-C4], all presupposed in the present work, is briefly reviewed in $\$ \S 1$ and 2 for the reader's convenience. The material in these two sections is independently known by G. Hector and G. Duminy and much of the terminology employed here and some other aspects of the presentation have been influenced by extensive conversations with them. These conversations have also been very helpful in the development and presentation of some of the other ideas in this paper.

A nonexponential, nonpolynomial leaf at finite level must belong to an open, saturated set $U$ of locally dense leaves without holonomy, the leaves in $U$ being mutually diffeomorphic and having a common growth type, (3.6) and (3.7). As proven in [C-C3, Proposition 1], the leaves in such a set $U$ either all have one locally dense end or they all have two such ends. In $\$ 6$, we will show that the nonpolynomial growth types can only occur when the leaves have one locally dense end. In the case of two locally dense ends, and also in both cases when the foliation is transversely analytic, the leaves in $U$ will have exactly polynomial growth of degree determined by the level of $U$ and by the group of periods (6.10). N. Tsuchiya has also obtained the transversely analytic case [Ts2].

Finally, in \$7, we will produce leaves at finite level having "fractional growth". Indeed, we will produce the growth type of $\gamma_{s}(m)=m^{s}$ for each real number $s>2$. Here we only prove that these foliations are of class $C^{1}$, but highly technical

Received by the editors July 27, 1979 and, in revised form, February 6, 1981.

1980 Mathematics Subject Classification. Primary 57R30; Secondary 58F18, 58H05, 57 R95.

${ }^{1}$ Partially supported by NSF Grant MCS-8001547.

2 Partially supported by NSF Grant MCS-8003248. 
considerations (discovered some time after the present paper was submitted) do allow our constructions to be $C^{\infty}$-smoothed [C-C5]. Again the leaves in question will appear in open, saturated sets without holonomy.

Conventions. All foliations will be transversely oriented of codimension one on closed manifolds and, except in $\S 7$, of class at least $C^{2}$.

The growth function $g_{L}: Z^{+} \rightarrow R^{+}$of a leaf $L$ will be defined, as in [P], via chains of placques from a suitable regular cover $\left\{W_{i}\right\}_{i=1}^{k}$. The growth type $\operatorname{gr}(L)=\operatorname{gr}\left(g_{L}\right)$ is defined as in [He1] via the equivalence relation of mutual dominance and is independent of all choices. By compactness of the ambient manifold, all growth functions are dominated by the exponential function.

We say that $\operatorname{gr}(L)$ is polynomial if $g_{L}$ is dominated by a polynomial. It is exactly polynomial of degree $k$ if $\operatorname{gr}\left(g_{L}\right)=\operatorname{gr}\left(m^{k}\right)$. It is quasipolynomial if $\lim _{m \rightarrow \infty}(1 / m) \log \left(g_{L}(m)\right)=0$ and nonexponential if $\lim _{\inf _{m \rightarrow \infty}}(1 / m) \log \left(g_{L}(m)\right)$ $=0$. Otherwise, $\operatorname{gr}(L)$ is exponential.

Let $M$ be the closed $n$-manifold with foliation $\mathscr{F}$ and fix a one-dimensional foliation $\mathcal{L}$ transverse to $\mathscr{F}$. Let $U \subset M$ be an open, connected, $\mathscr{F}$-saturated set. As in [D], let $\hat{U}$ denote the completion of $U$ in any Riemannian metric inherited from $M$, this being a manifold such that $\partial \hat{U}$ has finitely many components. The natural immersion $i: \hat{U} \rightarrow M$ is one-one on $U$ and on each component of $\partial \hat{U}$, carrying such a component onto a leaf of $\bar{U}-U$. We let $\delta U=i(\partial \hat{U})$ (the set of border leaves to $U$ ). Foliations $\hat{\mathscr{F}}$ and $\hat{\mathcal{L}}$ of $\hat{U}$ are induced by $\mathscr{F}$ and $\mathcal{L}$. By [D, Theorem 1], $\hat{U}$ is the union of a compact nucleus $K$, a manifold with corners, and finitely many complete, connected, noncompact "arms" $\hat{U}_{i} \cong B_{i} \times[-1,1], B_{i} \subset \partial \hat{U}$, the factors $\{x\} \times[-1,1]$ being leaves of $\hat{\mathrm{e}}$.

It will be useful to require that the regular cover $\left\{W_{i}\right\}_{i=1}^{k}$ of $M$, used in the definition of the growth functions, be biregular. This means that each $\bar{W}_{i} \cong D^{n-1}$ $\times[-1,1]$ in such a way that each $D^{n-1} \times\{t\}$ is a placque of $\mathscr{F}$ and each $\{x\} \times[-1,1]$ is a placque of $\mathcal{L}$. One can "intersect" $\overline{W_{i}}$ with $\hat{U}$ in an obvious way, producing (possibly infinitely many) components of the form $D^{n-1} \times[a, b],-1 \leqslant$ $a<b \leqslant 1$. The interiors, relative to $\hat{U}$, of these sets form a (generally infinite) biregular cover of $\hat{U}$, the growth of leaves of $\hat{\mathscr{F}}$ being defined via the placques of this cover.

1. The theory of levels. We summarize results from [C-C4] that provide the setting of the present paper.

Suppose that the foliation $\mathscr{F}$ of $M$ has smoothness class $C^{2}$. Let $U \subset M$ be an open, connected, $\mathscr{F}$-saturated set.

(1.1) Definition. Minimal sets of $\mathscr{F} \mid U$ are called local (or relative [D]) minimal sets of $\mathscr{F}$.

REMARK. If the foliation were smooth-leaved of class $C^{0}, \mathscr{F} \mid U$ might admit no minimal sets.

(1.2) Theorem [C-C4]. For $M, \mathscr{F}$, and $U$ as above, and for $L$ a leaf of $\mathscr{F} \mid U$, $\bar{L} \cap U$ contains a finite, nonzero number of minimal sets of $\mathscr{F} \mid U$. 
A proper leaf of $\mathscr{F}$ is always a local minimal set. Open, $\mathscr{F}$-saturated, connected sets $U \subset M$ are local minimal sets if each leaf of $\mathscr{F} \mid U$ is dense in $U$ (these are said to be of locally dense type). All other local minimal sets meet the leaves of $\mathcal{E}$ in sets homeomorphic to open subsets of the Cantor set (and are said to be of exceptional type).

Evidently, minimal sets of $\mathcal{F}$ are also local minimal sets. One merely takes $U=M$.

(1.3) Definition. A minimal set of $\mathscr{F}$ (and each of its leaves) is said to be at level 0 . A local minimal set $X$ of $\mathscr{F}$ (and each of its leaves) is at level $k>0$ if the highest level of any local minimal set in $\bar{X}-X$ is $k-1$. A leaf of $\mathscr{F}$ that is not at any level $k \geqslant 0$ is said to be at infinite level.

(1.4) THEOREM [C-C4]. For each integer $k \geqslant 0$, the union $M_{k}$ of all leaves of $\mathcal{F}$ at levels at most $k$ is compact. Furthermore, for each leaf $L$ of $\mathscr{F}$ and each $k \geqslant 0$, $\bar{L} \cap M_{k}$ is a finite, nonempty union of local minimal sets.

(1.5) THEOREM [C-C4]. Let $L$ be a leaf of $\mathcal{F}$ at infinite level. Then, for each integer $k \geqslant 0, \bar{L} \cap\left(M_{k}-M_{k-1}\right) \neq \varnothing$ (taking $\left.M_{-1}=\varnothing\right)$. If $X=\cup_{k \geqslant 0} \bar{L} \cap M_{k}$ and $Z=\bar{L}-X$, then

(1) $X$ is dense in $\bar{L}$;

(2) $Z$ is an uncountable union of leaves;

(3) each leaf of $Z$ is dense in $\bar{L}$;

(4) no leaf of $Z$ has a proper side.

From this it follows, in particular, that every local minimal set is at finite level.

In this paper we will be particularly interested in local minimal sets $U$ of locally dense type such that $\mathscr{F} \mid U$ is without holonomy. In this case, Theorem 4 of [Sa] allows us to construct a transverse, continuous, positive, invariant measure $\mu$ for $\mathscr{F} \mid U$, finite on compact subarcs of $\mathcal{L} \mid U$ (see Conventions). By [D, Theorem 3], the holonomy at every boundary leaf of $\hat{U}$ is unbounded; hence $\mu$ grows without bound near $\partial \hat{U}$. Thus, exactly as in [C-C3, Proposition 3] one proves

(1.6) Proposition. There is a $C^{0}$-flow $\Phi: \mathbf{R} \times M \rightarrow M$, nonsingular precisely on $U$, carrying the leaves of $\mathcal{F}$ diffeomorphically onto one another, the flow lines in $U$ being the leaves of $\mathcal{L} \mid U$.

(1.7) Definition. The group $P(\mu)$ of periods of $\mu$ is the additive group of real numbers $r$ such that $\Phi_{r}$ carries each leaf of $\mathscr{F} \mid U$ to itself.

2. The Poincaré-Bendixson theory of totally proper leaves. We continue our review of certain results from [C-C4]. The assumptions on $M$ and $\mathscr{F}$ continue to be as in $\$ 1$.

(2.1) Definition. Let $L$ be a leaf of $\mathscr{F}$. The substructure of $L$, denoted by $S(L)$, is the union of all leaves $F \subset \bar{L}$ such that $\bar{F} \neq \bar{L}$.

Equivalently, $S(L)$ is the union of all leaves of $\bar{L}$ of level strictly less than the level of $L$. Evidently, $S(L)$ is a union of local minimal sets, none of which is of the locally dense type. 
(2.2) Definition. If each leaf of $S(L)$ is proper, then $L$ is said to have totally proper substructure. If, in addition, $L$ itself is proper, then $L$ is said to be a totally proper leaf.

We will see (3.4) that nonexponential leaves have totally proper substructure. Here we outline the structure theory of totally proper leaves.

A leaf $L$ of $\mathcal{F}$ is a complete Riemannian $(n-1)$-manifold in any metric relativized from $M$. Let $\left\{B_{i}\right\}_{i>0}$ be a collection of complete, noncompact, connected, $(n-1)$-dimensional submanifolds of $L$, each with compact boundary. If $B_{0} \supset B_{1} \supset \cdots \supset B_{i} \supset \ldots$ and if $\bigcap_{i>0} B_{i}=\varnothing$, then $\left\{B_{i}\right\}_{i>0}$ defines an end $\varepsilon$ of $L$ and is said to be a fundamental system of (closed) neighborhoods of $\varepsilon$. For quick reviews of the facts and definitions concerning ends of open manifolds, see [C-C1], [C-C2], [C-C3]. For details, see [A-S], [F]. Let $\mathcal{E}(L)$ denote the set of ends of $L$, a compact, totally disconnected, metrizable space. For $\varepsilon \in \mathcal{E}(L)$ and $\left\{B_{i}\right\}_{i>0}$ as above, we define the $\varepsilon$-limit set of $L$ to be

$$
\varepsilon-\lim (L)=\bigcap_{i>0} \overline{B_{i}}
$$

where $\bar{B}_{i}$ denotes the closure of $B_{i}$ in $M$. This generalizes the $\alpha$ - and $\omega$-limit sets of classical Poincaré-Bendixson theory. The set $\varepsilon-\lim (L)$ is a closed, $\mathscr{F}$-saturated set, not depending on the choice of fundamental neighborhood system of $\varepsilon$.

(2.3) Definition. If $\varepsilon-\lim (L)$ is a union of totally proper leaves, then $\varepsilon$ is said to be a totally proper end of $L$.

If an end $\varepsilon$ of $L$ is totally proper, then the system $\left\{B_{i}\right\}_{i>0}$ can be chosen in an especially nice way, each $B_{i}$ "spiraling in" on a certain totally proper leaf $F$.

In order to describe this efficiently, we make use of the 1-dimensional foliation $\mathcal{L}$ (see Conventions). By a choice of transverse orientation of $\mathcal{F}$, each leaf of $\mathcal{L}$ is oriented. Subarcs of leaves of $\mathcal{L}$, therefore, will be denoted by standard interval notation $([x, y],[x, y)$, etc.).

On one side of $F$ (for definiteness, say the positive side) fix a half-open collar neighborhood $W$, fibered over $F$ by subarcs $[x, y), x \in F$, of leaves of $\ell$. Let $p$ : $W \rightarrow F$, where $p(z)=x$ if $z \in[x, y)$.

Let $B_{0} \subset L$ be a complete, connected, noncompact $(n-1)$-dimensional submanifold, $\partial B_{0}=N_{0}$ connected and compact.

(2.4) Definition. We will say that $B_{0}$ spirals on $F$ if the following conditions are satisfied.

(a) $B_{0} \subset W$; hence $p: B_{0} \rightarrow F$ is well defined and, on $B_{0}-N_{0}$, this is a local diffeomorphism.

(b) $p$ maps $N_{0}$ diffeomorphically onto $N \subset F$ and $F-N$ is connected.

(c) $p^{-1}(N)$ is a disjoint union of countably many diffeomorphic copies of $N$ : $N_{0}, N_{1}, N_{2}, \ldots$

(d) The connected components $B_{00}, B_{01}, B_{02}, \ldots$ of $B_{0}-\cup_{i>0} N_{i}$ are each mapped by $p$ diffeomorphically onto $F-N$ and, if $B_{0 j}^{c}$ denotes the closure of $B_{0 j}$ in $L$, then $\partial B_{0 j}^{c}=N_{j} \cup N_{j+1}, j \geqslant 0$.

(e) For each $x \in F$, the sequence $p^{-1}(x)=\left\{x_{j}=\left(B_{0 j} \cup N_{j}\right) \cap p^{-1}(x)\right\}_{j>0}$ converges monotonically to $x$. 
In the above situation, we take $B_{i}=\cup_{j>i} B_{0 j}^{c}$, getting a fundamental system of closed neighborhoods of an end $\varepsilon \in \mathcal{E}(L)$, where each $B_{i}$ spirals on $F$ and $\partial B_{i}=N_{i}, i \geqslant 0$. In this situation, $\varepsilon-\lim (L)=\bar{F}$ and $F$ is at strictly lower level than $L$.

(2.5) Definition. The end $\varepsilon \in \mathcal{E}(L)$ as above is said to be periodic of period $F$. The following pictures suggest some simple examples.

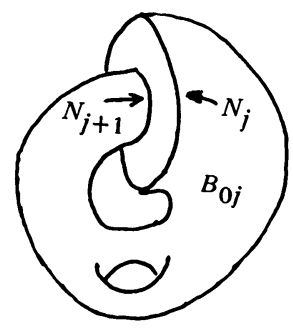

$$
B_{0}=\underbrace{\xi}_{N_{0} \sigma_{B_{00}} \underbrace{\sigma_{B_{01}}}_{N_{1}} \underbrace{\sigma_{N_{3}}}_{B_{02}}} \rightarrow
$$

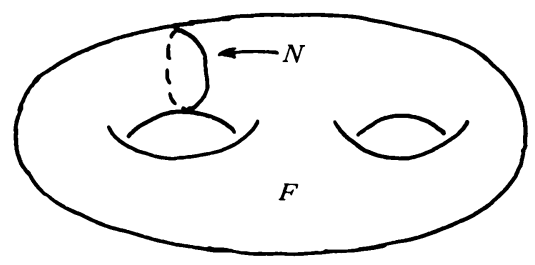

Figure A
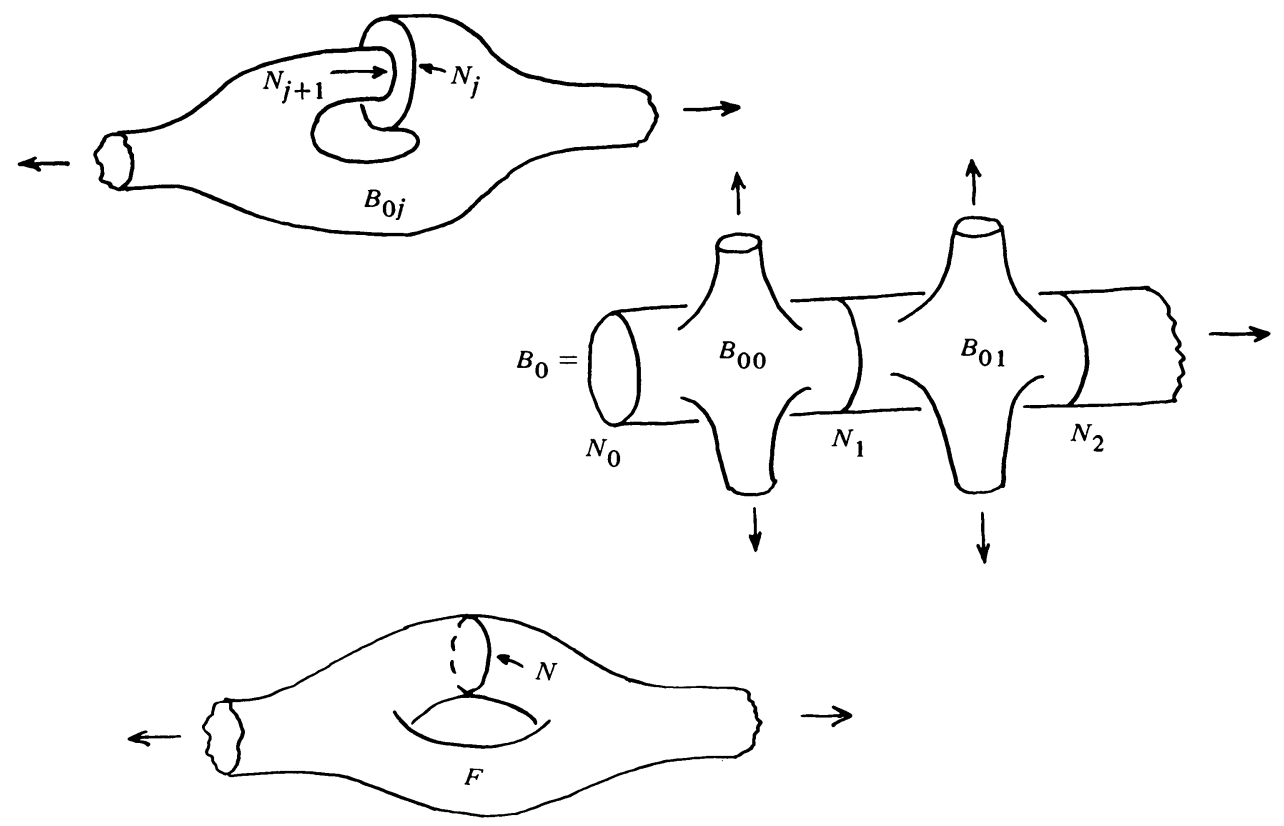

Figure B 
In Figure $\mathrm{A}$, the periodic end $\varepsilon$ is isolated in $\mathcal{E}(L)$. In Figure $\mathrm{B}, \varepsilon$ is the limit of a sequence of isolated ends in $\mathcal{E}(L)$.

(2.6) THEOREM [C-C4]. If $\varepsilon$ is a totally proper end of $L$, then $\varepsilon-\lim (L)=\bar{F}$ and $\varepsilon$ is periodic of period $F$.

If $L$ itself is totally proper, then each of its ends satisfies (2.6). Although generally there are infinitely many ends in $\mathcal{E}(L)$, it is not hard to use (2.6) to produce the following finite (nonunique) decomposition of a totally proper leaf $L$.

(2.7) Corollary. Let $L$ be a totally proper leaf of $\mathcal{F}$ at level $k$. Then $L=A \cup B^{1}$ $\cup \cdots \cup B^{r}$ where

(a) $A$ is a compact, connected, $(n-1)$-dimensional submanfold of $L$;

(b) each $B^{i}$ is a complete, connected, noncompact, $(n-1)$-dimensional submanifold of 1 ;

(c) $B^{i} \cap B^{j}=\varnothing, i \neq j$, and $A \cap B^{i}=\partial B^{i}$ is a component of $\partial A, 1 \leqslant i \leqslant r$;

(d) each $B^{i}$ spirals on a (totally proper) leaf $F^{i}$ of level at most $k-1$;

(e) at least one $F^{i}$ is at level $k-1$.

It is elementary that, if $F^{i}$ has growth function $g(m)$, then $B^{i}$ has the growth type of the function $m g(m)$. Since compact leaves have exactly polynomial growth of degree 0 , an obvious induction on the level of $L$, using (2.7), gives the following.

(2.8) COROLlary. Let $L$ be a totally proper leaf of $\mathscr{F}$ at level $k$. Then $L$ has exactly polynomial growth of degree $k$.

Finally, we remark on a topological corollary of (2.7). Recall ([C-C1], [C-C2], [C-C3]) that $L$ is said to be a manifold of (finite) type $q$ if the $q$ th derived set $\mathcal{E}^{(q)}(L) \subset \mathcal{E}(L)$ is finite and nonempty. If $L$ is compact (i.e., $\mathcal{E}(L)=\varnothing$ ), we say that $L$ is of type -1 . An induction on the level of $L$, using (2.7), gives the following.

(2.9) Corollary. Let $L$ be a totally proper leaf of $\mathscr{F}$ at level $k$. Then $L$ is a manifold of type $k-1$.

3. Nonexponential leaves. Following [L], we place the following definition. All notations and conventions are as in $\$ 2$.

(3.1) Definition. Let $L$ be a leaf of $\mathscr{F}, x \in L, J=[x, y)$ of $(y, x]$ a subarc of a leaf of $L$, and suppose that a suitable loop on $L$ at $x$ defines a holonomy contraction $g: J \rightarrow J$ to $x$. If $L \cap \operatorname{int}(J) \neq \varnothing$, then $L$ is said to be a resilient leaf.

The following fundamental result will be proven by an argument due essentially to J. F. Plante. Also, cf. [He2].

(3.2) THEOREM. Let $F$ be a leaf of $\mathscr{F}$ and suppose that $\bar{F}$ contains a resilient leaf. Then $F$ has exponential growth.

Proof. Let $L \subset \bar{F}$ be a resilient leaf and let $[x, y)$ and $g$ be as in (3.1). Since there is a point $z \in L \cap(x, y)$ and since $L \subset \bar{F}, F$ meets $(x, y)$. Because of $g$, $F \cap[x, y)$ clusters at $x$. By [P, Theorem 4.1], if $F$ has nonexponential growth, there 
is a holonomy invariant measure $\mu$ on $[x, z]$, with $\mu[x, z]=1$ and $\operatorname{supp}(\mu) \subset \bar{F} \cap$ $[x, z]$. But $\{x\}=\bigcap_{m \geqslant 0} g^{m}[x, z]$, so $\mu(\{x\})=1$. Since $\mu$ is invariant under the holonomy pseudogroup of $\mathscr{F}, \mu(\{z\})=1$. But then $x<\cdots<g^{m+1}(z)<g^{m}(z)$ $<\cdots<z$ and $\mu\left(\left\{g^{m}(z)\right\}\right)=1, \forall m \geqslant 0$, contradicting $\mu[x, z]=1$.

As observed in [C-C3], the proof of Sacksteder's well-known theorem [Sa, Theorem 1] actually gives the following. Also, cf. G. Hector's thesis.

(3.3) THEOREM. Let $X$ be a local minimal set of $\mathcal{F}$ of exceptional type. Then there is a resilient leaf in $X$.

REMARKS. For (3.3), the requirement that $\mathcal{F}$ be of class $C^{2}$ is essential (the $C^{1}$-counterexample being Denjoy's construction [Sc, Appendix]). For (3.2), it is enough that $\mathscr{F}$ be of class $C^{0}$.

(3.4) COROLlaRY. If $L$ is a leaf of $\mathscr{F}$ with nonexponential growth, then $L$ has totally proper substructure.

Proof. The substructure $S(L)$ is a union of local minimal sets, none of which is of locally dense type. If any is of exceptional type, then (3.3) and (3.2) contradict the assumption that $L$ is nonexceptional.

(3.5) COROLlaRY. If $L$ has a proper side (we say that $L$ is semiproper) and has nonexponential growth, then $L$ is totally proper.

Proof. By part (4) of (1.5), $L$ is at finite level, hence belongs to a local minimal set. This cannot be of locally dense type nor, by (3.3) and (3.2), can it be exceptional. Thus, $L$ is proper; hence, by (3.4), $L$ is totally proper.

Consequently, by $\S 2$,

(3.6) CoROllaRy. A semiproper, nonexponential leaf of $\mathcal{F}$ at finite level $k \geqslant 0$ must have exactly polynomial growth of degree $k$. It must also be a manifold of type $k-1$.

(3.7) THEOREM. Let $L$ be a nonexponential leaf of $\mathcal{F}$ at finite level. If $L$ does not have a proper side, then $L \subset U$ where $U$ is a local minimal set of locally dense type, $\mathscr{F} \mid U$ has trivial holonomy, and the leaves of $\mathscr{F} \mid U$ are mutually diffeomorphic and have the same growth type as $L$. If $L$ is at level $k$, then it is a manifold of type at most $k$. Finally, $\bar{U}-U$ is a finite union of totally proper leaves at levels at most $k-1$, at least one of which is at level $k-1$.

Proof. Since $L$ is not proper and cannot pertain to a local minimal set of exceptional type (again by (3.3) and (3.2)), it must pertain to a local minimal set $U$ of locally dense type. The transverse, holonomy invariant measure of Plante [P], defined on any transverse circle to $\mathscr{F} \mid U$, replaces [Sa, Theorem 4] in the proof of (1.6), so we obtain $\Phi: \mathbf{R} \times M \rightarrow M$ as in that proposition. Each $\Phi_{t}, t \in \mathbf{R}$, transforms any given biregular cover of $M$ (see Conventions) to another such. It follows that the growth types of the leaves of $\mathscr{F} \mid U$, being determined by such covers, are all the same. The argument in [C-C3, Theorem 4] shows that the leaves of $\mathscr{F} \mid U$ are manifolds of type at most $k$. Since $\bar{U}-U=S(L)$, the final assertion follows from (3.4). 
Remark that, by (3.6) and (3.7), any nonexponential, non-exactly-polynomial leaf at finite level must pertain to an open, $\mathscr{F}$-saturated set $U$ as described in (3.7).

4. Some abstract growth estimates. We assemble here some technical results that will be useful for $\S 5$.

Fix a sequence $1 \leqslant N(1)<N(2)<\cdots<N(k)<\cdots$ of integers and let $A \geqslant 1$ be an integer. We define three increasing functions $\mathbf{Z}^{+} \rightarrow \mathbf{R}^{+}$:

$$
\begin{aligned}
f^{*}(m) & =m^{k} / k !, & N(k-1)<m \leqslant N(k), \\
f^{* *}(m) & =m^{k} / k !, & A N(k-1)<m \leqslant A N(k), \\
f^{* * *}(m) & =m^{k} A^{k} / k !, & N(k-1)<m \leqslant N(k) .
\end{aligned}
$$

Here we allow all $k \geqslant 1$ by taking $N(0)=0$.

(4.1) Proposition. $\operatorname{gr}\left(f^{*}\right)=\operatorname{gr}\left(f^{* *}\right)=\operatorname{gr}\left(f^{* * *}\right)$.

Proof. Clearly, $f^{* * *}(m) \geqslant f^{*}(m) \geqslant f^{* *}(m)$, for all $m$. If $A N(k-1)<A m \leqslant$ $A N(k)$, then $N(k-1)<m \leqslant N(k)$, so $f^{* *}(A m)=(A m)^{k} / k !=f^{* * *}(m)$. Thus, $\operatorname{gr}\left(f^{* * *}\right) \geqslant \operatorname{gr}\left(f^{*}\right) \geqslant \operatorname{gr}\left(f^{* *}\right) \geqslant \operatorname{gr}\left(f^{* * *}\right)$.

For each $k \geqslant 1$, let $\mathcal{G}_{k} \subset \mathbf{R}^{k}$ be the graph with vertices the integer lattice $\mathbf{Z}^{k}$ and edges all line segments of length one, parallel to one or another coordinate axis, with endpoints in $\mathbf{Z}^{k}$. This, of course, is just a convenient geometric realization of the graph of the group $\mathbf{Z}^{k}$ relative to the standard generating set. The growth function $f_{k}(m)$ is the number of vertices of $\mathcal{S}_{k}$ that can be reached from the origin by paths in $\mathcal{G}_{k}$ of length at most $m$. Define

$$
f(m)=f_{k}(m), \quad N(k-1)<m \leqslant N(k) .
$$

(4.2) Proposition. $\operatorname{gr}(f)=\operatorname{gr}\left(f^{*}\right)$.

This will be proven via a series of elementary lemmas ((4.3) through (4.9)).

(4.3) LEMMA. If $a>1$ and if $p$ is a positive integer, then $(a-1)^{p} \geqslant a^{p}-p a^{p-1}$.

Indeed, for $a \leqslant p$, the right-hand side is nonpositive and, for $a>p$, the binomial theorem gives the result.

(4.4) Lemma. For all integers $m \geqslant 1$ and $k \geqslant 1, \sum_{i=0}^{m} i^{k} \geqslant m^{k+1} /(k+1)$.

Proof. This is true for $m=1$. If it is true for some $m$, then

$$
\sum_{i=0}^{m+1} i^{k} \geqslant(m+1)^{k}+m^{k+1} /(k+1) .
$$

In (4.3), take $a=m+1$ and $p=k+1$ and obtain $m^{k+1} \geqslant(m+1)^{k+1}-$ $(k+1)(m+1)^{k}$. Then

$$
(m+1)^{k}+m^{k+1} /(k+1) \geqslant(m+1)^{k+1} /(k+1) .
$$

(4.5) LEMMA. $f_{k}(m) \geqslant m^{k} / k$ !

Proof. It is clear that $f_{1}(m)=2 m+1 \geqslant m$. We use induction on $k$. If the assertion holds for some $k \geqslant 1$, then by (4.4) 


$$
f_{k+1}(m) \geqslant \sum_{i=0}^{m} f_{k}(i) \geqslant \sum_{i=0}^{m} i^{k} / k ! \geqslant m^{k+1} /(k+1) ! .
$$

(4.6) LemMA. For each $m \geqslant 1$ and $k \geqslant 1, \sum_{i=0}^{m-1} i^{k} \leqslant m^{k+1} /(k+1)$.

PRoof. This is clearly true for $m=1$. If it is true for some $m \geqslant 1$, then

$$
\sum_{i=0}^{m} i^{k} \leqslant m^{k}+m^{k+1} /(k+1)=\left(m^{k+1}+(k+1) m^{k}\right) /(k+1) .
$$

But the binomial theorem implies that $m^{k+1}+(k+1) m^{k} \leqslant(m+1)^{k+1}$.

For the next three lemmas, let $\tilde{f}_{k}(m)$ denote the number of vertices in $\mathcal{G}_{k}$, with all coordinates nonnegative, that can be reached by paths from 0 of length at most $m$. Remark that $f_{k}(m) \leqslant 2^{k} \tilde{f}_{k}(m)$.

(4.7) LeMmA. For each $m \geqslant 1$ and $k \geqslant 1, \tilde{f}_{k}(m) \leqslant(m+k)^{k} / k$ !.

Proof. Since $\tilde{f}_{1}(m)=m+1$, the assertion is true for $k=1$. Inductively, assume it true for some $k \geqslant 1$. Then (letting $\tilde{f}_{k}(0)=1 \leqslant k^{k} / k$ !) we have $\tilde{f}_{k+1}(m)=$ $\sum_{i=0}^{m} \tilde{f}_{k}(i) \leqslant(1 / k !) \sum_{i=0}^{m}(i+k)^{k} \leqslant(1 / k !) \sum_{j=0}^{m+k} j^{k} \leqslant(m+k+1)^{k+1} / k !(k+1)$ $=(m+k+1)^{k+1} /(k+1)$ !. This last inequality is an application of (4.6).

(4.8) LEMMA. If $m \geqslant k \geqslant 1$, then $\tilde{f}_{k}(m) \leqslant 4^{k} m^{k} / k$ !.

Proof. If $m \geqslant 1$, then $\tilde{f}_{1}(m)=m+1 \leqslant 4 m$, so we assume, inductively, that the assertion is true for some $k \geqslant 1$. If $m \geqslant k+1$, then $\tilde{f}_{k+1}(m)=\sum_{i=0}^{m} \tilde{f}_{k}(i)=$ $\sum_{i=0}^{k} \tilde{f}_{k}(i)+\sum_{i=k+1}^{m-1} \tilde{f}_{k}(i)+\tilde{f}_{k}(m)$ and

$$
\begin{aligned}
\sum_{i=0}^{k} \tilde{f}_{k}(i) & \leqslant \sum_{i=0}^{k}(i+k)^{k} / k ! \leqslant \sum_{i=0}^{2 k} i^{k} / k ! \leqslant(2 k+1)^{k+1} /(k+1) ! \\
& \leqslant 2^{k+1}(k+1)^{k+1} /(k+1) ! \leqslant 4^{k} m^{k+1} /(k+1) !
\end{aligned}
$$

Also,

$$
\begin{gathered}
\sum_{i=k+1}^{m-1} \tilde{f}_{k}(i) \leqslant \sum_{i=0}^{m-1} 4^{k} i^{k} / k ! \leqslant 4^{k} m^{k+1} /(k+1) !, \\
\tilde{f}_{k}(m) \leqslant 4^{k} m^{k} / k ! \leqslant 4^{k} m^{k+1} /(k+1) !
\end{gathered}
$$

(the last inequality also uses $m \geqslant k+1)$. Thus, $\tilde{f}_{k+1}(m) \leqslant 3\left(4^{k} m^{k+1} /(k+1)\right.$ !) $\leqslant$ $4^{k+1} m^{k+1} /(k+1)$ !.

(4.9) LeMma. If $m \geqslant k$, then $f_{k}(m) \leqslant 8^{k} m^{k} / k$ !.

PRoof. Indeed, $f_{k}(m) \leqslant 2^{k} \tilde{f}_{k}(m) \leqslant 8^{k} m^{k} / k$ ! by (4.8).

We prove (4.2). By (4.5), $f_{k}(m) \geqslant m^{k} / k !$, so $\operatorname{gr}(f) \geqslant \operatorname{gr}\left(f^{*}\right)$. Since $\{N(i)\}_{i>1}$ is a strictly increasing sequence of positive integers, $N(k-1)<m \leqslant N(k)$ implies, in particular, that $m \geqslant k$, so $f_{k}(m) \leqslant 8^{k} m^{k} / k$ ! (4.9). In the definition of $f^{* * *}$, take $A=8$ and conclude that $\operatorname{gr}(f) \leqslant \operatorname{gr}\left(f^{* * *}\right)=\operatorname{gr}\left(f^{*}\right)$. This completes the proof of (4.2).

Using (4.2), we will determine $\operatorname{gr}(f)$ for particular choices of $\{N(k)\}_{k>1}$. The following will be useful. 
(4.10) Lemma. If $B \geqslant 1$ is an integer, then $B f^{*}(m) \leqslant f^{*}(B m)$.

Proof. Let $i \leqslant j$ be such that

$$
\begin{aligned}
f^{*}(B m) & =(B m)^{j} / j !, \quad N(j-1)<B m \leqslant N(j), \\
B f^{*}(m) & =B m^{i} / i !, \quad N(i-1)<m \leqslant N(i) .
\end{aligned}
$$

Since $N(j-1) \geqslant j-1$, we have $B m \geqslant j$, so $(B m / j)^{j-i} \geqslant 1$. But

$$
(B m)^{j-i} / j(j-1) \cdots(i+1) \geqslant(B m / j)^{j-i} \geqslant 1,
$$

so

$$
f^{*}(B m)=(B m)^{j} / j ! \geqslant(B m)^{i} / i ! \geqslant B m^{i} / i !=B f^{*}(m) .
$$

A. A continuum of quasipolynomial but nonpolynomial growth types. For each real number $r>1$, let $N_{r}(k)=\left[k^{r}\right]$, the greatest integer in $k^{r}$. With this choice of $\left\{N(k)=N_{r}(k)\right\}_{k \geqslant 1}$, denote the corresponding functions $f$ and $f^{*}$ by $f_{(r)}$ and $f_{(r)}^{*}$.

(4.11) Proposition. If $s>r>1$, then $\operatorname{gr}\left(f_{(r)}\right)>\operatorname{gr}\left(f_{(s)}\right)$.

Proof. By (4.2), it is sufficient to prove that $\operatorname{gr}\left(f_{(r)}^{*}\right)>\operatorname{gr}\left(f_{(s)}^{*}\right)$. If $N_{s}(k-1)<m$ $\leqslant N_{s}(k)$, then $f_{(s)}^{*}(m)=m^{k} / k$ ! while, since $\left.N_{r}(k-1)<N_{s}(k-1), f_{(r)}^{*}(m)\right\rangle$ $m^{k} / k !=f_{(s)}^{*}(m)$. Thus, $\operatorname{gr}\left(f_{(r)}^{*}\right) \geqslant \operatorname{gr}\left(f_{(s)}^{*}\right)$. To make the inequality strict, we show that given positive integers $m_{0}, A, B$ there exists $m \geqslant m_{0}$ such that $B f_{(s)}^{*}(A m)<$ $f_{(r)}^{*}(m)$. Choose $K$ so large that, for $k \geqslant K$,

(i) $A B N_{r}(2 k)<N_{s}(k)$,

(ii) $m_{0} \leqslant N_{r}(2 k)$,

(iii) $A B<N_{s}(k)-N_{s}(k-1)$,

(iv) $2 k A B<N_{r}(2 k)$.

For such $k$, (iii) allows us to choose a largest value of $m$ such that $N_{s}(k-1)<$ $A B m \leqslant N_{s}(k)$. By (i) and (ii), $m_{0} \leqslant N_{r}(2 k) \leqslant m$. Furthermore, $f_{(s)}^{*}(A B m)=$ $(A B m)^{k} / k$ ! and $f_{(r)}^{*}(m)=m^{q} / q$ ! where $q \geqslant 2 k$. Also, $m>N_{r}(q-1) \geqslant q-1$, so $m \geqslant q$ and

$$
f_{(r)}^{*}(m)=\frac{m^{q-2 k}}{q(q-1) \cdots(2 k+1)} \frac{m^{2 k}}{(2 k) !} \geqslant \frac{m^{2 k}}{(2 k) !} .
$$

By (iv), $2 k A B<N_{r}(2 k) \leqslant m$. Thus, $(2 k A B)^{k}<m^{k}$ so $((2 k) ! / k !)(A B m)^{k}<m^{2 k}$; hence $(A B)^{k} m^{k} / k !<m^{2 k} /(2 k)$ !. Thus, by (4.10) and the above, $B f_{(s)}^{*}(A m) \leqslant$ $f_{(s)}^{*}(A B m)<f_{(r)}^{*}(m)$.

(4.12) Proposition. For each $r>1, f_{(r)}$ has quasipolynomial but nonpolynomial growth.

Proof. If $N_{r}(k-1)<m \leqslant N_{r}(k)$, then $k<m^{1 / r}+1$. Thus,

$$
\begin{aligned}
f_{(r)}^{*}(m) & =m^{k} / k ! \geqslant(m / k)^{k}>\left(m /\left(m^{1 / r}+1\right)\right)^{k} \\
& =\left(m^{1-1 / r} /\left(1+m^{-1 / r}\right)\right)^{k}>\left(\frac{1}{2} m^{1-1 / r}\right)^{k} .
\end{aligned}
$$

Since $r$ is fixed and $k$ increases with $m$, it follows that $f_{(r)}^{*}$ dominates polynomials of every degree. That is, $\operatorname{gr}\left(f_{(r)}\right)=\operatorname{gr}\left(f_{(r)}^{*}\right)$ is nonpolynomial. 
For $m$ in the above range, $f_{(r)}(m)=f_{k}(m) \leqslant(2 m+1)^{k}$, so

$$
\begin{aligned}
(1 / m) \log \left(f_{k}(m)\right) & \leqslant(k / m) \log (2 m+1)<\left(\left(m^{1 / r}+1\right) / m\right) \log (2 m+1) \\
& =\left(1+m^{-1 / r}\right)(\log (2 m+1)) / m^{1-1 / r} \leqslant\left(2 / m^{1-1 / r}\right) \log (2 m+1) .
\end{aligned}
$$

Since $r>1$, L'Hôpital's rule gives $\lim _{m \rightarrow \infty}(1 / m) \log \left(f_{(r)}(m)\right)=0$.

REMARKs. (1) Using Sterling's formula [R, p. 194], one can show that $\operatorname{gr}\left(f_{(r)}^{*}\right)=$ $\operatorname{gr}\left(f_{(r)}^{\#}\right)$ where $f_{(r)}^{\#}(m)=m^{k} / k^{k}, N_{r}(k-1)<m \leqslant N_{r}(k)$.

(2) Using the above remark, one can show that $\operatorname{gr}\left(f_{(r)}\right)=\operatorname{gr}\left(g_{r}\right)$ where $g_{r}(m)=$ $m^{\left(m^{1 / \prime}\right)}$

B. A continuum of nonquasipolynomial growth types. The following is immediate.

(4.13) LEMMA. If, for some $k \geqslant 1, N(k+1)=N(k)+1=m+1$, then $f(m+1)$ $\geqslant 2 f(m)$.

(4.14) Corollary. If $N(k)=k$ for all $k \geqslant 1$, then $f$ dominates the exponential function.

Proof. In (4.13), $m=k=N(k)$ for all $m \geqslant 1$, so $f(m+1) \geqslant 2^{m} f(1)$.

Combining (4.13) with the proof of (4.12), we produce a sequence $\left\{\bar{N}_{r}(k)\right\}_{k>1}$ so that the corresponding function $f=\bar{f}_{(r)}$ has nonexponential but nonquasipolynomial growth. We will then show that distinct values of $r>1$ will give distinct growth types $\operatorname{gr}\left(\bar{f}_{(r)}\right)$.

Let $\{k(q)\}_{q>1}$ be a strictly increasing sequence of integers, $k(1) \geqslant 2$, and set $\bar{N}_{r}(k(q))=\left[(k(q))^{r}\right], \bar{N}_{r}(k(q)+i)=\bar{N}_{r}(k(q))+i$ for $1 \leqslant i \leqslant k(q+1)-k(q)-$ 1 .

(4.15) LEMMA. If $\{k(q)\}_{q \geqslant 1}$ increases fast enough, then $\operatorname{gr}\left(\bar{f}_{(r)}\right)$ is nonexponential but nonquasipolynomial.

Proof. Set $m(q)=\bar{N}_{r}(k(q))+1$. Then we have $\bar{N}_{r}(k(q))<m(q) \leqslant \bar{N}_{r}(k(q)+1)$ and the proof of (4.12) shows that $\lim _{q \rightarrow \infty}(1 / m(q)) \log \left(\bar{f}_{(r)}(m(q))\right)=0$, so $\operatorname{gr}\left(\bar{f}_{(r)}\right)$ is nonexponential.

Suppose $k(q)$ has been chosen and choose $k(q+1)$ sufficiently large as follows. Repeated applications of (4.13) give $\bar{f}_{(r)}(m(q)+i) \geqslant 2 i \bar{f}_{(r)}(m(q))$, provided $k(q+1)$ $-2 \geqslant k(q)+i$. Thus,

$$
(1 /(m(q)+i)) \log \left(\bar{f}_{r}(m(q)+i)\right) \geqslant(i /(m(q)+i)) \log (2) .
$$

As $i$ becomes large, the right-hand side approaches $\log (2)$. Thus, for a sufficiently large choice of $k(q+1)$, taking $i=k(q+1)-k(q)-2$ makes the left-hand side greater than $\frac{1}{2} \log (2)$. By such an inductive choice, we guarantee that $(1 / m) \log \left(\bar{f}_{(r)}(m)\right)$ does not converge to 0 .

(4.16) Proposition. If $s>r>1$, then $\operatorname{gr}\left(\bar{f}_{(r)}\right) \neq \operatorname{gr}\left(\bar{f}_{(s)}\right)$.

Proof. Let the corresponding functions $f^{*}$ be denoted $\bar{f}_{(r)}^{*}$ and $\bar{f}_{(s)}^{*}$ respectively. If $\bar{f}_{(s)}^{*}$ does not dominate $\bar{f}_{(r)}^{*}$, these functions cannot have the same growth type. Thus, given positive integers $m_{0}, A, B$, we must show that there exists an integer $m \geqslant m_{0}$ such that $B \bar{f}_{(s)}^{*}(A m)<\bar{f}_{(r)}^{*}(m)$. Choose $K$ as in the proof of (4.11) and $k>K$ such 
that $\bar{N}_{s}(k)=\left[k^{s}\right]=N_{s}(k)$. Also choose $m$ as in the proof of (4.11). Then we claim that

$$
B \bar{f}_{(s)}^{*}(A m) \leqslant \bar{f}_{(s)}^{*}(A B m)=f_{(s)}^{*}(A B m)<f_{(r)}^{*}(m) \leqslant \bar{f}_{(r)}^{*}(m) .
$$

The first inequality is by (4.10). The equality holds because $\bar{N}_{s}(k-1) \leqslant N_{s}(k-1)$ $<A B m \leqslant N_{s}(k)=\bar{N}_{s}(k)$ (as in the proof of (4.11)). The strict inequality is proven as in the proof of (4.11) and the last inequality is clear.

5. Constructions of nonexponential leaves at finite level. Let $\mathbf{G}$ be the set of growth types of the functions $m f_{(r)}(m), m \bar{f}_{(r)}(m)$, and $e^{m}$, all $r>1$. Here the notation is as in $\S 4$. Thus, $\mathbf{G}$ contains a continuous infinity of distinct quasipolynomial but nonpolynomial types ((4.11) and (4.12)) and a continuum of distinct nonexponential but nonquasipolynomial types ((4.15) and (4.16)), together with the exponential type.

Our first aim is a constructive proof of the following.

(5.1) Theorem. Let $M$ be a closed 3-manifold and let $\gamma \in \mathbf{G}$. Then there is a $C^{\infty}$-foliation $\mathscr{F}$ of $M$ containing a local minimal set $U$ of locally dense type such that

(a) $\bar{U}-U$ is a finite union of totally proper leaves;

(b) $\mathscr{F} \mid U$ has trivial holonomy, the leaves being mutually diffeomorphic;

(c) each leaf of $\mathscr{F} \mid U$ has the growth type $\gamma$.

REMARKS. (1) It is an easy exercise to adapt the construction so as to produce exactly polynomial growth of degree $k \geqslant 3$ in $\mathscr{F} \mid U$.

(2) While (3.6) and (3.7) predicted that a nonexponential but nonpolynomial leaf at finite level would lie in a set $U$ as above, our theorem shows that a leaf in such a set can also have exponential growth.

(3) We could have employed exactly the same computations as are found in [He1] rather than those of $\$ 4$. But the computations in [He1], being intended for other purposes, are somewhat more complicated than what we need here.

Let $T$ be the closed, orientable surface of genus 2 and let $I=[-1,1]$. Let $h, l \in \operatorname{Diff}_{+}^{\infty}(I)$ and suppose they are $C^{\infty}$-flat at \pm 1 . One obtains a $C^{\infty}$-foliation $\mathscr{F}(h, l)$ of $T \times I, C^{\infty}$-trivial at the boundary, as follows. First foliate $T \times I$ by the leaves $T \times\{t\}$, then cut this open along the annuli $C_{1} \times I$ and $C_{2} \times I\left(C_{1}\right.$ and $C_{2}$ are the circles on $T$ pictured in Figure $C)$ and reglue via the identifications $(x, t) \equiv(x, h(t)), x \in C_{1}$, and $(y, t) \equiv(y, l(t)), y \in C_{2}$.

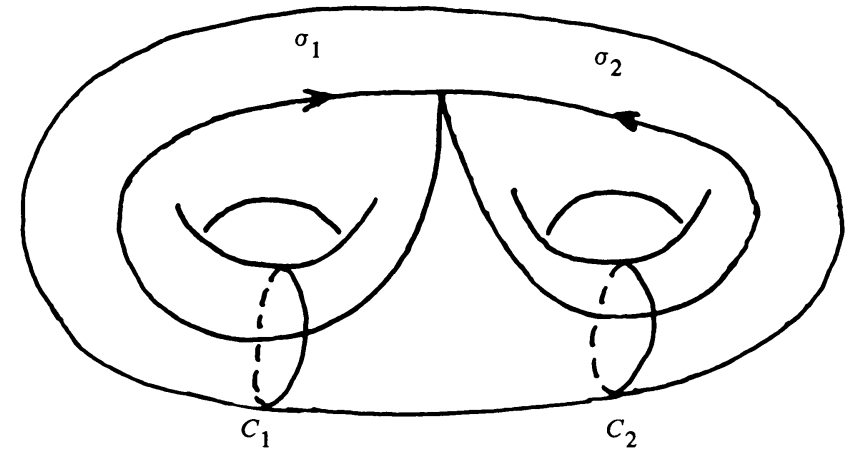

Figure C 
Let $L_{t}$ denote the leaf through $\left(x_{0}, t\right)$. Let $G \subset \operatorname{Diff}_{+}^{\infty}(I)$ denote the group generated by $\{h, l\}$. Then, if we identify $\left\{x_{0}\right\} \times I$ with $I$, the leaf $L_{t}$ meets $I$ exactly in the trajectory $G(t)$. The lifts of $\sigma_{1}$ and $\sigma_{2}$ (see Figure C) become the edges of an imbedded graph $|G(t)| \subset L_{t}$ with vertices the set $G(t)$. This graph is isomorphic to the usual graph of the trajectory $G(t)$ relative to the generators $\{h, l\}$. Let $g: \mathbf{Z}^{+} \rightarrow \mathbf{R}^{+}$denote the growth function, based at $t$, of this graph. That is, $g(m)$ is the number of vertices reached from $t$ by paths in $|G(t)|$ consisting of at most $m$ edges. It is well known and elementary that the growth type of $g$ coincides with that of the leaf $L_{t}$.

Remark that many lifts of $\sigma_{2}$ are loops. Deleting these gives a graph $|G(t)|^{*}$ with the same growth function $g$.

Let $\mathbf{G}^{*}$ consist of the growth types of $f_{(r)}(m), \bar{f}_{(r)}(m)$, and $e^{m}$, all $r>1$.

(5.2) Claim. For the proof of (5.1), it is sufficient, for each $\gamma \in \mathbf{G}^{*}$, to produce $\mathscr{F}(h, l)$ as above having a saturated set $U$ with the properties listed in (5.1).

Proof. Imbed $T \times I \subset S^{2} \times S^{1}$ so that some fiber $\left\{x_{0}\right\} \times S^{1}$ meets $T \times I$ in the fiber $\left\{x_{0}\right\} \times I$. Writing $S^{1}$ as a union $I \cup J$ of compact arcs meeting at their common boundary $\{ \pm 1\}$, we use the method of Thurston [Th] to foliate $S^{2} \times S^{1}$ - int $(T \times I)$ so that the boundary components are leaves, the foliation is $C^{\infty}$-trivial at the boundary, and $\left\{x_{0}\right\} \times J$ is a transverse arc. The fact that Thurston's theorem applies follows from elementary topological considerations as in [G]. The condition on $\left\{x_{0}\right\} \times J$ is easy to guarantee in Thurston's construction simply by making sure that $\left\{x_{0}\right\} \times J$ is a union of 1-simplices in the counter-oriented fine triangulation that he employs. Instead of Thurston's theorem, one can also use more elementary constructions from [C-C1]. One completes this to a foliation $\mathscr{F}$ of $S^{2} \times S^{1}$ by foliating $T \times I$ with $\mathscr{F}(h, l)$. Then $\sigma=\left\{x_{0}\right\} \times S^{1}$ is a closed transversal to $\mathscr{F}$ and one performs the standard modification along $\sigma$ and removes the interior of the new Reeb component. This produces a foliation of the solid torus, $C^{\infty}$-trivial at the boundary. In a standard way, again by Thurston's existence theorem [Th], this foliated solid torus can be incorporated as a component of suitable $C^{\infty}$-foliations of all closed 3-manifolds $M$.

If $U$ is a component in $\mathscr{F} \mid(T \times I)$ having the given properties, there results a new, open, saturated set $U^{*} \subset M, \bar{U}^{*}-U^{*}$ consisting of proper leaves of respective levels one higher than those of $\bar{U}-U$, together with one new toral leaf. If $\gamma=\operatorname{gr}(g(m))$ is the growth type of the leaves in $U$, then it is well known and elementary that $\gamma^{*}=\operatorname{gr}(m g(m))$ is the growth type of the leaves in $U^{*}$.

We choose $h$ so that $h(t)>t,-1<t<1$. Fix $[\bar{a}, a] \subset(-1,1)$ such that $h(\bar{a})=a$. Let $\varphi(t) d / d t$ be a smooth vector field on $I$ such that $\operatorname{supp}(\varphi)=[\bar{a}, a]$ and $\varphi(t)>0$ for $\bar{a}<t<a$. Let $\Phi: \mathbf{R} \times I \rightarrow I$ be the flow generated by this field.

Let $\{c(k)\}_{k \geqslant 1}$ be a strictly decreasing sequence of rationally independent, positive, real numbers converging to 0 . Let $\{N(k)\}_{k>1}$ be a strictly increasing sequence of positive integers. Define

$$
l_{k}=h^{N(k)} \circ \Phi_{c(k)} \circ h^{-N(k)},
$$

supported on $h^{N(k)}([\bar{a}, a])$. If $\{c(k)\}_{k \geqslant 1}$ converges to 0 rapidly enough, the infinite composition $l=\ldots \circ l_{k} \circ l_{k-1} \circ \ldots \circ l_{1}$ is a $C^{\infty}$-diffeomorphism of $I$ and is $C^{\infty}$-flat at \pm 1 . The following is elementary. 
(5.3) LEMMA. Let $\mathcal{F}=\mathscr{F}(h, l)$ and let $U$ be the $\mathscr{F}$-saturation of $(\bar{a}, a)$. Then each leaf of $\mathcal{F} \mid U$ is dense in $U$ and has trivial holonomy, and the only leaves in $T \times I-U$ are the proper leaves $T \times\{ \pm 1\}$ and $L_{a}$ at levels 0 and 1 respectively.

In $\S 4$ we defined $f: \mathbf{Z}^{+} \rightarrow \mathbf{R}^{+}$depending only on the choice of the sequence $\{N(k)\}_{k \geqslant 1}$.

(5.4) LEMMA. Each leaf of $\mathscr{F} \mid U$ has the growth type of $f$.

Proof. The natural inclusions $\mathcal{G}_{1} \subset \cdots \subset \mathcal{G}_{k} \subset \cdots$ of the graphs in $\$ 4$ give rise to a limit graph $\mathcal{G} \subset \mathbf{R}^{\infty}$. Because of the rational independence of $\{c(k)\}_{k>1}$ we can imbed $|G(t)|^{*} \subset \mathcal{G}$ in a natural way, taking $t$ to the origin $(0,0, \ldots) \in \mathcal{G}$. Indeed, given a vertex $v \in|G(t)|^{*}$, choose any path from $t$ to $v$ and note that the corresponding word in $h$ and $l$ can be viewed as a word in $h, l_{1}, \ldots, l_{r}$ for some $r \geqslant 0$. The vertex $v$ does not determine a unique such word, but it does determine the sum $y_{1}$ of the exponents of $h$, the sum $y_{2}$ of the exponents of $l_{1}$, etc. Thus $v$ corresponds to $\left(y_{1}, y_{2}, \ldots, y_{r}, 0,0, \ldots\right) \in \mathcal{G}$. This gives a one-one correspondence between the vertices of $|G(t)|^{*}$ and those of $\mathcal{G}$. Every edge in $\mathcal{G}$ parallel to the $x_{1}$-axis is also an edge in $|G(t)|^{*}$ corresponding to an action of $h$. If (and only if) $v$ has $x_{1}$-coordinate $y_{1}=N(k)$, then the edge in $\mathcal{G}$ coming out of $v$ and parallel to the $x_{k+1}$-axis is also an edge in $|G(t)|^{*}$ corresponding to an action of $l_{k}$.

For the proof of the lemma, we compare shortest paths in $|G(t)|^{*}$ to shortest paths in $\mathcal{G}$. A canonical choice of shortest paths in $|G(t)|^{*}$ can be fixed by the formula

$$
h^{\alpha(k)} \circ l_{k-1}^{\alpha(k-1)} \circ h^{N(k-1)-N(k-2)} \circ l_{k-2}^{\alpha(k-2)} \circ \cdots . h^{N(2)-N(1)} \circ l_{1}^{\alpha(1)} \circ h^{N(1)} .
$$

Here we assume $\alpha(k-1) \neq 0$ if $k>1$ and agree that the formula reduces to $h^{\alpha(1)}$ if $k=1$. This path is said to be of degree $k$. Note that it has length $r=N(k-1)$ $+\sum_{i=1}^{k}|\alpha(i)|$ (taking $N(0)=0$ ). The shortest path in $\mathcal{G}$ from the origin to the same vertex has length $q=|N(k-1)+\alpha(k)|+\sum_{i=1}^{k-1}|\alpha(i)|$. Note that $r \leqslant q+$ $2 N(k-1)$.

Let $g_{k}(m)$ denote the number of canonical paths of degree at most $k$ and length at most $m$. That is, $g_{k}$ is the growth function of $|G(t)|^{*} \cap \mathcal{G}_{k}$. From the above paragraph, it follows that, if $m^{*} \geqslant m+2 N(k-1)$, then $f_{k}(m) \leqslant g_{k}\left(m^{*}\right)$. Thus, if $N(k-1)<m \leqslant N(k)$, we obtain $f(m)=f_{k}(m) \leqslant g_{k}(3 m) \leqslant g(3 m)$. On the other hand, it is clear that, for $m$ in the same range, $g(m)=g_{k}(m) \leqslant f_{k}(m)=f(m)$.

By the results of $\S 4$, together with (5.2), (5.3), and (5.4), the proof of (5.1) is complete.

The group of periods. Let $U$ be a local minimal set of locally dense type such that $\bar{U}-U$ is totally proper and $\mathcal{F} \mid U$ has no holonomy. Let the transverse, invariant measure $\mu$, the $C^{0}$-flow $\Phi: \mathbf{R} \times M \rightarrow M$, and the group of periods $P(\mu)$ be as in $\S 1$ (cf. (1.6) and (1.7)). Remark that, in the above constructions, the group of periods is free abelian on the infinite generating set $\{c(k)\}_{k \geqslant 1}$.

In [P, Theorem 6.3] it was shown that, if $U=M$, then $P(\mu) \cong \mathbf{Z}^{p(\mu)}, 2 \leqslant p(\mu)$ $<\infty$, and each leaf of $\mathcal{F}$ has polynomial growth (in fact, exactly polynomial) of degree $p(\mu)-1$. An adaptation of this argument (also, cf. [He2, Theorem 4]) shows 
that, if $\bar{U}-U$ is a finite, nonempty union of compact leaves, then each leaf of $\mathscr{F} \mid U$ has exactly polynomial growth of degree $p(\mu)$, where $p(\mu)$ is again finite and $P(\mu)=Z^{p(\mu)}$. By (5.1), these results do not generalize to the case in which $\bar{U}-U$ is not a finite union of compact leaves. In fact, in this case there is little, if any, relation between the structure of the period group $P(\mu)$ and the growth in $\mathscr{F} \mid U$.

(5.5) TheOREM. Let $M$ be a closed 3-manifold and let $P \subset \mathbf{R}$ be a dense, finitely or countably generated, additive subgroup. For each of the following possibilities, there is $a C^{\infty}$-foliation $\mathcal{F}$ of $M$ having an open, $\mathcal{F}$-saturated set $U$ and transverse, invariant measure $\mu$, all as above, such that $P(\mu)=P$ and the common growth type in $\mathcal{F} \mid U$ is

(a) quasipolynomial but nonpolynomial; or

(b) nonexponential but not quasipolynomial; or

(c) exponential.

(5.6) Claim. For the proof of (5.5) it will be sufficient to produce a foliation $\mathcal{F}(h, l)$ of $T \times I$ having the required properties.

This is proven exactly as (5.2). One needs only note that the method in that proof for inserting the construction into an arbitrary 3-manifold will not change the group $P(\mu)$.

We emphasize that quite a variety of subgroups $P \subset \mathbf{R}$ satisfy the conditions in (5.5). Not only might $P \cong \mathbf{Z}^{p}, 2 \leqslant p<\infty$, or $P \cong \mathbf{Q}$, but $P$ can have any finite rank $p \geqslant 2$, yet be irreducible [Po, pp. 36-38], or it can have infinite rank. Nevertheless, the following is elementary.

(5.7) LEMMA. Given $P$ as above, there is a sequence $\{c(k)\}_{k \geqslant 2}$, decreasing strictly monotonically to 0 as rapidly as desired, such that $P$ is generated by this sequence.

There is no problem carrying out the previous constructions using the above sequence $\{c(k)\}_{k \geqslant 1}$ and any strictly increasing sequence $\{N(k)\}_{k \geqslant 1}$ of positive integers, but the growth estimates are harder because $\{c(k)\}_{k \geqslant 1}$ will not generally consist of rationally independent numbers. However, the weaker assertions in (5.5) will be easy to verify. As usual, the fact that $\mathscr{F}(h, l)$ is $C^{\infty}$ and is $C^{\infty}$-flat at $T \times\{ \pm 1\}$ is guaranteed by choosing $\{c(k)\}_{k \geqslant 1}$ to converge to 0 rapidly enough by (5.7).

Define $\hat{f}(m)=2^{k}, N(k-1)<m \leqslant N(k)$. If $g$ denotes the growth function of $|G(t)|$, then we claim that

(5.8) LEMMA. If $\{c(k)\}_{k \geqslant 1}$ is chosen to decrease rapidly enough then $\operatorname{gr}(\hat{f}) \leqslant \operatorname{gr}(g)$ $\leqslant \operatorname{gr}(f)$.

Proof. Every vertex in $|G(t)|^{*}$ can be reached by a shortest path from $t$ of the form given in the proof of (5.4). However, we can no longer claim that the vertex determines this path uniquely nor that every path of this form is shortest. Let $|\tilde{G}(t)|^{*}$ be determined by the same sequence $\{N(k)\}_{k>1}$ and a rationally independent sequence $\{\tilde{c}(k)\}_{k \geqslant 1}$. Then the growth function $\tilde{g}(m)$ of this graph counts the paths of length at most $m$ of the above form, so $g(m) \leqslant \tilde{g}(m)$. Thus, by (5.4), $\operatorname{gr}(g) \leqslant \operatorname{gr}(\tilde{g})=\operatorname{gr}(f)$. 
Make sure that $\{c(k)\}_{k \geqslant 1}$ decreases so fast that $c(m)>\sum_{k=m+1}^{\infty} c(k), \forall m \geqslant 1$. Then, in $|G(t)|^{*}$, paths at $t$ of the form

$$
l_{k-1} \circ h^{N(k-1)-N(k-2)} \circ l_{k-2}^{\varepsilon(k-2)} \circ \ldots \circ h^{N(2)-N(1)} \circ l_{1}^{e(1)} \circ h^{N(1)},
$$

all $\varepsilon(i) \in\{0,1\}$, correspond one-one to their endpoints and are of length $N(k-1)$ $+1+\sum_{i=1}^{k-2} \varepsilon(i) \leqslant 2 N(k-1)$. It follows that, if $N(k-1)<m \leqslant N(k)$, then $2^{k-2}$ $\leqslant g(2 m)$; hence $\hat{f}(m)=2^{k} \leqslant 4 g(2 m)$. This proves that $\operatorname{gr}(\hat{f}) \leqslant \operatorname{gr}(g)$.

We use (5.8) to prove (5.5). To obtain exponential growth, we choose $N(k)=k$, $k \geqslant 1$, and observe that $\hat{f}(m)=2^{m}$. For quasipolynomial growth, we choose $N(k)$ $=k^{2}, k \geqslant 1$. Then $\operatorname{gr}(g) \leqslant \operatorname{gr}(f)$ is quasipolynomial by (4.12). But also, $\hat{f}(m)=2^{k}$ $\geqslant 2^{\sqrt{m}},(k-1)^{2}<m \leqslant k^{2}$, so $\operatorname{gr}(g) \geqslant \operatorname{gr}(\hat{f})$ is nonpolynomial. Finally, as in the proof of (4.15), one judiciously chooses $N(k)=k^{2}$ for some values of $k$ and $N(k)=N(k-1)+1$ for the others. As in (4.15), $\operatorname{gr}(g) \leqslant \operatorname{gr}(f)$ is nonexponential. If $N(k)=N(k-1)+1$, then $\hat{f}(N(k))=2 \hat{f}(N(k-1))$, so it is easy to carry out the judicious choice so that $\operatorname{gr}(g) \geqslant \operatorname{gr}(\hat{f})$ is not quasipolynomial. This completes the proof of (5.5).

Of course, all of the constructions in this section provide examples in many closed manifolds of dimension $n>3$. Indeed, if $M^{n}$ factors into $M^{3} \times M^{n-3}$, our constructions in $M^{3}$ can simply be multiplied by $M^{n-3}$.

6. The case of two locally dense ends. Here the closed manifold $M$ is of arbitrary dimension $n \geqslant 2$ and the foliation $\mathcal{F}$ is of class $C^{2}$. We assume that $U \subset M$ is a local minimal set of locally dense type and that $\mathscr{F} \mid U$ has trivial holonomy. As usual, we obtain the transverse, invariant measure $\mu$ for $\mathscr{F} \mid U$ as in $\S 1$, the $C^{0}$-flow $\Phi: \mathbf{R} \times M \rightarrow M$ of (1.6), and the group $P(\mu)$ of periods (1.7).

Let $L$ be a leaf of $\mathcal{F} \mid U$. An end $\varepsilon \in \mathcal{E}(L)$ will be called locally dense if $\varepsilon-\lim (L)=\bar{U}$. The following was proven in [C-C3, Proposition 1].

(6.1) THEOREM. Either each leaf of $\mathscr{F} \mid U$ has one locally dense end or each leaf has two such ends.

The proof of (6.1) is analogous to H. Hopf's famous proof [Ho] that a regular covering space of a compact manifold has the same number of ends as the group of covering transformations (namely, one, two, or a Cantor set). Indeed, via a result of D. Tischler [Ti], (6.1) is a direct consequence of Hopf's theorem in the case $U=M$, but, for the general case, examples show that such a reduction to [Ho] is impossible for multiple reasons.

In the case $U=M$, one finds that $P(\mu) \cong \mathbf{Z}^{p}, 2 \leqslant p<\infty$, and that the case of two (locally) dense ends corresponds exactly to $p=2$. For the general case this also fails. Indeed, one can have $\hat{U} \cong T^{2} \times[-1,1]$ with $\mathscr{F} \mid U$ a foliation by dense planes. In this case $P(\mu) \cong \mathbf{Z} \times \mathbf{Z}$, but the leaves have one locally dense end.

Finally, in the case $U=M$, the leaves of $\mathscr{F}$ have polynomial growth (in fact, exactly polynomial) of degree $p-1$, where $P(\mu) \cong \mathbf{Z}^{p}$. If $\hat{U}$ is compact, one again gets $P(\mu) \cong \mathbf{Z}^{p}, 2 \leqslant p<\infty$, and the growth in $\mathscr{F} \mid U$ is exactly polynomial of degree $p$. For these facts, cf. [P, Theorem 6.3], [He 2, Theorem 4]. But the constructions in $\$ 5$ show that this too fails to generalize further. 
However, we will establish the following.

(6.2) THEOREM. Suppose that the leaves of $\mathscr{F} \mid U$ have two locally dense ends and that $\bar{U}-U$ is totally proper. Let $d$ be the highest level of any leaf in $\bar{U}-U(d=-1$ if $\bar{U}-U=\varnothing$ ). Then $P(\mu) \cong \mathbf{Z} \times \mathbf{Z}$ and each leaf of $\mathscr{F} \mid U$ has exactly polynomial growth of degree $d+2$.

In particular, the exotic growth types produced in $\$ 5$ force the leaves to have only one locally dense end.

Our first goal will be to prove that $P(\mu) \cong \mathbf{Z} \times \mathbf{Z}$. Let $S$ be a closed transversal to $\mathscr{F} \mid U$ and arrange that this be a leaf of $\mathcal{L}$. This is easy to do by modifying the tangent field to any given choice of $\mathcal{E}$ in a small tubular neighborhood of $S$. Let $L$ be a leaf of $\mathscr{F} \mid U$ and let $\alpha$ and $\omega$ be the locally dense ends of $L$. It is easy to find a compact, connected submanifold $N_{0} \subset L$ of codimension one such that $L-N_{0}$ has two components, $W_{0}$ and $V_{0}$, neighborhoods of $\alpha$ and $\omega$ respectively. It will be convenient to select $N_{0}$ such that $S \cap N_{0} \neq \varnothing$.

Any homeomorphism $\varphi: L \rightarrow L$ extends uniquely to a homeomorphism (again denoted $\varphi$ ) of the compactification $L \cup \mathcal{E}(L)$ onto itself. In particular, if $t \in P(\mu)$, then $\Phi_{2 t}: L \rightarrow L$ so extends and permutes the set $\{\alpha, \omega\}$. The same, of course, is true of $\Phi_{t}$; hence $\Phi_{2 t}=\Phi_{t}^{2}$ must be the identity on $\{\alpha, \omega\}$.

For $t$ as above, let $N_{1}=\Phi_{2 t}\left(N_{0}\right)$. If $t \neq 0$ and is small enough, it is clear that $N_{1} \cap N_{0}=\varnothing$. Replacing $t$ with its negative, if necessary, we assume $N_{1} \subset W_{0}$. Also, $W_{1}=\Phi_{2 t}\left(W_{0}\right)$ is the component of $L-N_{1}$ that is a neighborhood of $\alpha$, so $W_{1} \subset W_{0}$. Thus, $B_{0}=\left(N_{0} \cup W_{0}\right)-W_{1}$ is a complete submanifold of $L$ with $\partial B_{0}=N_{0} \cup N_{1}$, and $\mathcal{E}\left(B_{0}\right) \subset \mathcal{E}(L)$ consists of ends that are not locally dense (hence, every end of $B_{0}$ is a totally proper end of $L$ ).

An iteration is now possible, producing $N_{k}=\Phi_{2 k t}\left(N_{0}\right), B_{k}=\Phi_{2 k t}\left(B_{0}\right), W_{k}=$ $\Phi_{2 k t}\left(W_{0}\right)$, and $V_{k}=\Phi_{2 k t}\left(V_{0}\right), k \in \mathbf{Z}$. The manifolds $B_{k}$ are complete, they have disjoint interiors, $\partial B_{k}=N_{k} \cup N_{k+1}$, and $\mathcal{E}\left(B_{k}\right)$ is a set of totally proper ends of $L$. The manifolds $N_{k}$ are also disjoint.

The principal step in the computation of $P(\mu)$ will be to choose $t \in P(\mu)$ as above, but so as to guarantee that $L=\cup_{k \in \mathbf{Z}} B_{k}$. Equivalently, $\left\{W_{k}\right\}_{k>0}$ and $\left\{V_{k}\right\}_{k<0}$ will be fundamental neighborhood systems of $\alpha$ and $\omega$ respectively.

First we point out an important consequence of the construction so far. Let $s=\mu(S)$, an element of $P(\mu)$.

(6.3) LEMMA. All sufficiently small, nonzero elements $t \in P(\mu)$ are rationally independent of $s$.

Proof. Take $x_{0} \in S \cap N_{0}$. The elements $x_{k}=\Phi_{2 k t}\left(x_{0}\right), k \in \mathbf{Z}$, are distinct points of $S$ since the manifolds $N_{k}$ are disjoint. Thus $2 k t$ is not an integral multiple of $s$ for any integer $k$.

Let $t_{0}, t_{1} \in P(\mu)$ both satisfy the above conditions on $t$. Because of (6.3), we can (and do) select $t_{1}$ rationally independent of $t_{0}$. Let $H \subset P(\mu)$ be the subgroup generated by $t_{0}$ and $t_{1}$. 
(6.4) LeMMA. Let $W \subset L$ be a neighborhood of $\alpha$ (respectively, of $\omega$ ). Then $\exists r \in H$ such that $\Phi_{2 r}\left(N_{0}\right) \subset W$.

Proof. The group $H$ is dense in $\mathbf{R}$. Choose a sequence $\left\{r_{i}\right\} \subset H$ of nonzero elements converging to 0 and such that each $r_{i}$ satisfies our usual conditions. Then $\Phi_{2 r_{i}}\left(N_{0}\right) \subset W_{0}$ and, in $\hat{U}, \Phi_{2 r_{i}}\left(N_{0}\right)$ converges uniformly to $N_{0}$ as $i \rightarrow \infty$. We emphasize that this convergence is relative to the measure $\mu$ along the leaves of $\hat{\mathcal{L}}$, not in $L$. It follows that, in $L \cup \mathcal{E}(L),\left\{\Phi_{2 r_{i}}\left(N_{0}\right)\right\}$ clusters at an end $\varepsilon$ such that $L \subset \varepsilon-\lim (L)$. That is, $\varepsilon$ is a locally dense end having $W_{0}$ as a neighborhood, so $\varepsilon=\alpha$. For suitable values of $i, \Phi_{2 r_{i}}\left(N_{0}\right) \subset W$, so we take $r=r_{i}$. If $W$ is a neighborhood of $\omega$, we argue in the same way, using the sequence $\left\{-r_{i}\right\}$.

(6.5) LEMMA. Let $W$ and $r \in H$ be as in (6.4). Let $W_{*}$ be the component of $W-\Phi_{2 r}\left(N_{0}\right)$ that is a neighborhood of $\alpha$ (respectively, of $\omega$ ). Then $\Phi_{2 r+2 t_{0}}\left(N_{0}\right)$ and $\Phi_{2 r+2 t_{1}}\left(N_{0}\right)$ (respectively, $\Phi_{2 r-2 t_{0}}\left(N_{0}\right)$ and $\Phi_{2 r-2 t_{1}}\left(N_{0}\right)$ ) both lie in $W_{*}$.

Proof. Indeed, $W_{*}=\Phi_{2 r}\left(W_{0}\right)$ (respectively, $W_{*}=\Phi_{2 r}\left(V_{0}\right)$ ) and $\Phi_{2 t_{0}}\left(N_{0}\right) \cup$ $\Phi_{2 t_{1}}\left(N_{0}\right) \subset W_{0}$ (respectively, $\left.\Phi_{-2 t_{0}}\left(N_{0}\right) \cup \Phi_{-2 t_{1}}\left(N_{0}\right) \subset V_{0}\right)$.

In our basic construction, take $t=t_{0}+t_{1}$. Thus, $N_{k}=\Phi_{2 k\left(t_{0}+t_{1}\right)}\left(N_{0}\right)$, etc.

(6.6) Corollary. In $L \cup \mathcal{E}(L), \lim _{k \rightarrow \infty}\left(N_{k}\right)=\alpha$ and $\lim _{k \rightarrow-\infty}\left(N_{k}\right)=\omega$.

Proof. Let $W \subset L$ be a neighborhood of $\alpha$. Choose $r \in H$ such that $\Phi_{2 r}\left(N_{0}\right) \subset$ $W$. Let $r=m_{0} t_{0}+m_{1} t_{1}$. If $m_{0} \leqslant m_{1}$, iterate (6.5) to conclude that, for $k \geqslant m_{1}$, $N_{k} \subset W$. Similarly, if $m_{1} \leqslant m_{0}$, we take $k \geqslant m_{0}$ to get $N_{k} \subset W$. This proves that $\lim _{k \rightarrow-\infty}\left(N_{k}\right)=\alpha$ and a similar argument gives $\lim _{k \rightarrow \infty}\left(N_{k}\right)=\omega$.

The structure of $P(\mu)$. We are ready to prove that $P(\mu) \cong \mathbf{Z} \times \mathbf{Z}$. By (6.6), $\cap_{k \geqslant 0} W_{k}=\varnothing$ and $\cap_{k \leqslant 0} V_{k}=\varnothing$, so $L=\cup_{k \in \mathbf{Z}} B_{k}$. The set $S \cap B_{0}$ must be finite. Otherwise it has an accumulation point on $S$, implying that a subsequence converges, in $B_{0} \cup \mathcal{E}\left(B_{0}\right)$, to an end $\varepsilon$ such that $\varepsilon-\lim (L)$ contains a leaf of $\mathscr{F} \mid U$. That is, $\varepsilon \in \mathcal{E}\left(B_{0}\right)$ would be a locally dense end, and this is impossible. We have supposed there is $x_{0} \in S \cap N_{0}$, so there are finitely many numbers $0=r_{0}$, $r_{1}, \ldots, r_{q}$ such that $S \cap\left(B_{0}-N_{1}\right)=\left\{\Phi_{r_{i}}\left(x_{0}\right)\right\}_{0 \leqslant i \leqslant q}$. Since $\Phi_{s} \mid S$ is the identity, and since $r \in P(\mu)$ if and only if $\Phi_{r}\left(x_{0}\right) \in S \cap L$, it follows that $s$ and $t$ generate a subgroup of rank 2 and index $q+1$ in $P(\mu)$. Since $P(\mu) \subset \mathbf{R}$, it follows that $P(\mu) \cong \mathbf{Z} \times \mathbf{Z}$.

(6.7) Proposition. Along each leaf of $\partial \hat{U}$, the holonomy is generated by a single contraction.

Proof. Let $F$ be a leaf of $\partial \hat{U}$. Assume $U$ lies on the positive side of $F$. By [D, Theorem 3], the holonomy along $F$ is unbounded, so the usual argument, using the measure $\mu$, shows the existence of a holonomy contraction along $F$. Let $x \in F$, $[x, y)$ a subarc of the leaf of $\hat{\mathcal{E}}$ containing $x$, and let $\sigma, \tau$ be loops on $F$ based at $x$ and defining contractions $h_{\sigma}, h_{\tau}:[x, y) \rightarrow[x, y)$. Since $\mu\left[h_{\sigma}(z), z\right]$ and $\mu\left[h_{\tau}(z), z\right]$ are elements of $P(\mu) \cong \mathbf{Z} \times \mathbf{Z}$, our assertion will follow if we show that suitable nonzero powers of these contractions are equal.

Let $\Gamma$ be the pseudogroup on $[x, z] \subset[x, y)$ generated by $h_{\sigma}$ and $h_{\tau}$. If these contractions are independent, then $\Gamma(z)$ is dense in $[x, z], \forall z \in(x, y)$. 
As in the proof of (6.4), choose a sequence $\left\{r_{i}\right\} \subset P(\mu)$ of nonzero elements converging to 0 and such that $\left\{N_{i}^{*}=\Phi_{2 r_{i}}\left(N_{0}\right)\right\}$ converges monotonically to $\alpha$. That is, $W_{1}^{*} \supset W_{2}^{*} \supset \ldots, \cap W_{i}^{*}=\varnothing$, where $W_{i}^{*}=\Phi_{2 r_{i}}\left(W_{0}\right)$ is the component of $L-N_{i}^{*}$ corresponding to $\alpha$. In $\hat{U}$, the manifolds $N_{i}^{*}$ are confined to a compact neighborhood of $N_{0}$; hence we can assume that there is a collar neighborhood $C$ of $F$ in $\hat{U}$ such that $N_{i}^{*} \cap C=\varnothing, \forall i \geqslant 1$. Let $B_{i}^{*}$ be the complete, connected submanifold of $L$ with $\partial B_{i}^{*}=N_{i}^{*} \cup N_{i+1}^{*}$. Then $\mathcal{E}\left(B_{i}^{*}\right)$ is a set of periodic ends of $L$ (2.6) and $\cup_{i \geqslant 1} B_{i}^{*}$ is a neighborhood of the locally dense end $\alpha$. Thus, for $i$ sufficiently large, we can find $z \in B_{i}^{*} \cap[x, y)$ so near to $x$ that all lifts of $\sigma$ and $\tau$ starting in $[x, z]$ and staying on leaves must stay in $C$. Since neither $N_{i}^{*}$ nor $N_{i+1}^{*}$ meets $C, \Gamma(z) \subset B_{i}^{*} \cap[x, z]$. If $\Gamma(z)$ were dense in $[x, z]$, some end in $\mathcal{E}\left(B_{i}^{*}\right)$ would be locally dense, giving a contradiction.

(6.8) Definition. If the nucleus $K \subset \hat{U}$ can be chosen so that, in each arm $\hat{U}_{j} \cong B_{j} \times[-1,1], \hat{\mathscr{F}}$ restricts to to the product foliation by leaves $B_{j} \times\{t\}$, then $\hat{\mathscr{F}}$ is said to be almost trivial.

The property defined in (6.8) makes sense (and often is verified) whether the leaves of $\mathscr{F} \mid U$ have one or two locally dense ends. However,

(6.9) Proposition. If the leaves of $\mathscr{F} \mid U$ have two locally dense ends, then $\hat{\mathcal{F}}$ is almost trivial.

Proof. By the proof of (6.7), together with (2.6), each leaf $F$ in $\partial \hat{U}$ must be the period of a periodic end $\varepsilon$ of $L$, and the basic contraction of (6.7) corresponds to the spiraling on $F$ of a neighborhood $B$ of $\varepsilon$ (2.4). Let $N \subset F$ be as in (2.4). If $\tau$ is a loop on $F$ at $x$ having empty intersection with $N$, the holonomy $h_{\tau}:[x, y) \rightarrow[x, y)$ fixes the points of $B \cap[x, y)$. Since $\mathscr{F} \mid U$ is without holonomy, $h_{\tau}$ must be the identity. Pick the nucleus $K$ of $\hat{U}$ large enough that $N \subset K \cap F$, and do this for every leaf $F$ of $\partial \hat{U}$. Then $\hat{\mathscr{F}}$ must induce the product foliation in each arm $\hat{U}_{i}$ of $\hat{U}-K$.

The following will complete the proof of (6.2). It has some interest in its own right. We do not assume two locally dense ends.

(6.10) Proposition. If $\hat{\mathscr{F}}$ is almost trivial, then $P(\mu) \cong \mathbf{Z}^{p(\mu)}, 2 \leqslant p(\mu)<\infty$. If also $\bar{U}-U$ is totally proper and $d$ is as in (6.2), the leaves of $\mathcal{F} \mid U$ have exactly polynomial growth of degree $p(\mu)+d$.

Indeed, $P(\mu)$ is the (everywhere dense) image of a homomorphism $\mu_{*}: H_{1}(U) \rightarrow$ $\mathbf{R}$ defined as follows. If $[\sigma] \in H_{1}(U)$, we represent it by a cycle $\sigma=\sum c_{i}$, each $c_{i}$ a singular 1-simplex either lying on a leaf of $\mathscr{F} \mid U$ or transverse to $\mathscr{F} \mid U$. If $c_{i}$ lies on a leaf, let $\mu_{\sharp}\left(c_{i}\right)=0$. If $c_{i}$ is transverse, $\mu_{\sharp}\left(c_{i}\right)= \pm \mu\left(c_{i}\right)$, the sign being + if and only if $c_{i}$ is directed in the positive sense of the transverse orientation of $\mathscr{F}$. Then $\mu_{*}[\sigma]=\Sigma \mu_{\sharp}\left(c_{i}\right)$ (cf. [P]). Since $\mathcal{F} \mid U$ induces a trivial foliation outside of $K \cap U$, $\mu_{*}$ vanishes out there; hence $P(\mu)$ is the image of $\mu_{*}: H_{1}(K \cap U) \rightarrow \mathbf{R}$. But $H_{1}(K \cap U)=H_{1}(K)$ and this is finitely generated; hence $P(\mu)$ is a finitely generated, dense subgroup of $\mathbf{R}$. This proves that $P(\mu) \cong \mathbf{Z}^{p(\mu)}, 2 \leqslant p(\mu)<\infty$.

For the rest of the proof of (6.10), we must specify a number of technical structures. We assume $\bar{U}-U$ is totally proper. 
Let $F_{1}, \ldots, F_{q}$ be the leaves of $\partial \hat{U}$ and remark that at least one of these leaves, say $F_{1}$, is at the maximal level $d$. Thus, $\operatorname{gr}\left(F_{1}\right)$ is exactly polynomial of degree $d$ and, generally, $\operatorname{gr}\left(F_{j}\right)$ is exactly polynomial of degree at most $d, 1 \leqslant j \leqslant q$ (cf. (2.8)).

Write $F_{j}=A_{j} \cup B_{j}^{1} \cup \cdots \cup B_{j}^{r}$, a decomposition as in (2.7), this and the nucleus $K$ being chosen so that $A_{j}=K \cap F_{j}, 1 \leqslant j \leqslant q$. Let $B_{j}^{i}(\hat{\mathcal{E}})$ denote the $\hat{\mathfrak{L}}$-saturation of $B_{j}^{i}$, this being an arm of $\hat{U}$ relative to $K$. The foliation $\hat{\mathcal{F}}$ induces $\hat{\mathscr{F}}_{j}^{i}$ on $B_{j}^{i}(\hat{\mathfrak{E}})$, this being the product foliation with leaves diffeomorphic to $B_{j}^{i}$ and having the same growth type as $B_{j}^{i}$ (exactly polynomial of degree at most $d$, cf. remarks preceding (2.8)). Let $N_{j}^{i} \subset L_{j}$ denote the interface of $B_{j}^{i}$ and $A_{j}$. The $\hat{\mathfrak{L}}$-saturation $N_{j}^{i}(\hat{\mathfrak{E}})$ is a face of the nucleus $K$.

Let $V_{1}, \ldots, V_{\lambda}$ be closed biregular neighborhoods (cf. Conventions) such that $K=V_{1} \cup \cdots \cup V_{\lambda}$. We can assume that a small tubular neighborhood $T_{j}^{i}$ of $N_{j}^{i}$ in $L_{j}$ has $\hat{\mathfrak{L}}$-saturation $V_{j}^{i}=T_{j}^{i}(\hat{\mathfrak{E}}) \cong T_{j}^{i} \times[-1,1]$, each $\{x\} \times[-1,1]$ being a leaf of $\hat{\mathcal{L}}$ and each $T_{j}^{i} \times\{t\}$ lying in a leaf of $\hat{\mathscr{F}}$. Let $J_{i}$ be a leaf of $\hat{\mathscr{L}} \mid V_{i}$ and $J_{j}^{i}$ a leaf of $\hat{\mathcal{E}} \mid V_{j}^{i}$. Fix $x \in \operatorname{int}\left(J_{1}\right)$ and let $L$ be the leaf of $\mathscr{F} \mid U$ through $x$. Define $\nu_{i}(m)$ (respectively $\nu_{j}^{i}(m)$ ) to be the number of distinct points of $J_{i}$ (respectively, of $J_{j}^{i}$ ) that can be reached by following a chain of $\mathscr{F}$-placques of length at most $m+1$. Here, of course, the placques in question come from the various $V_{i}$ and $V_{j}^{i}$, the latter placques being diffeomorphic to $T_{j}^{i}$.

Because each $\hat{\mathscr{F}}_{j}^{i}$ is trivial, $L \cap K$ is connected and has growth function the sum of all $\nu_{i}$ and $\nu_{j}^{i}$.

(6.11) LeMmA. If $J_{i} \subset U$, then $\nu_{i}$ has exactly polynmial growth of degree $p(\mu)-1$. Otherwise, $\nu_{i}$ (and each $\left.v_{j}^{i}\right)$ has exactly polynomial growth of degree $p(\mu)$.

Proof. Suppose that neither end of $J_{i}$ meets $\partial \hat{U}$. Then, exactly as in $[\mathbf{P}$, Theorem 6.3], one shows that $\nu_{i}$ is dominated by a polynomial of degree $p(\mu)-1$ (here we work in $K$ rather than in the manifold $M$ as in [P]). In the course of this proof, one sets $2 \delta=\mu\left(J_{i}\right)$ and considers the function $g(m)$ that counts the number of elements of $P(\mu) \cap[-\delta, \delta]$ that can be expressed as words of length at most $m$ in a fixed basis of $P(\mu)$. The crucial observation is that $g$ is dominated by a polynomial of degree $p(\mu)-1$. If an end of $J_{i}$ lies on $\partial \hat{U}$, then $[-\delta, \delta]$ is replaced by a (possibly half-) infinite interval and the degree of growth becomes $p(\mu)$. Thus, in this case, $\nu_{i}$ is dominated by a polynomial of degree $p(\mu)$, as is each $\nu_{j}^{i}$. Here we will show that, in each of these cases, the functions in question also dominate polynomials of these same degrees.

Fix $J=J_{i}$ or $J_{j}^{i}$. Let $\left\{t_{1}, \ldots, t_{p(\mu)}\right\}$ be a rationally independent subset of $P(\mu)$. If $J \subset U$, we lose no generality in assuming that both ends of $J$ lie in a common leaf; hence we can take $t_{p(\mu)}=\mu(J)$ in that case. If an end of $J$ lies on a leaf $F$ of $\partial \hat{U}$, let $\gamma$ be a holonomy contraction (6.7) on $J$ defined by a loop on $F$, take $y \in J \cap \operatorname{dom}(\gamma)$, and let $t_{p(\mu)}=\mu[\gamma(y), y]$ (where, for definiteness, we have assumed $\gamma(y)<y)$. Finally, arrange that $0<t_{i}<t_{p(\mu)}, 1 \leqslant i \leqslant p(\mu)-1$. Let $\Gamma$ denote the holonomy pseudogroup on $J$ defined by the foliation of $K$. 
First consider the case in which $J \subset U$. For each $z \in J$ and $1 \leqslant j \leqslant p(\mu)-1$, either $\Phi_{t_{j}}(z) \in J$ or $\Phi_{t_{j}-t_{p(\mu)}}(z) \in J$. There are corresponding elements $\gamma_{j 1}, \ldots, \gamma_{j k(j)}$ $\in \Gamma$, each with domain an open subset of $J$, and each coinciding with $\Phi_{t j}$ or $\Phi_{t_{j}-t_{p(\mu)}}$ on that domain, such that $J$ is covered by these domains. In this way one obtains a finitely generated subpseudogroup $\Gamma_{0} \subset \Gamma$ which, at each point of $J$, has growth dominating a polynomial of degree $p(\mu)-1$. Thus, in this case $J=J_{i}$ and $\nu_{i}$ has exactly polynomial growth of degree $p(\mu)-1$.

Next, consider the case in which $J$ has at least one end in $\partial \hat{U}$. Let $[\gamma(y), y] \subset J$ be as above. Let $\Gamma_{0}$ be the finitely generated pseudogroup on $[\gamma(y), y]$ defined as in the previous paragraph. Of course, $\Gamma_{0}$ can be considered as a pseudogroup on $J$; hence $\Gamma_{0} \subset \Gamma$. A new finitely generated subpseudogroup $\tilde{\Gamma}_{0} \subset \Gamma$ is defined by augmenting the generating set of $\Gamma_{0}$ by $\gamma$. At each point of $[\gamma(y), y]$ the growth of $\tilde{\Gamma}_{0}$ dominates a polynomial of degree $p(\mu)$. It follows that $J=J_{i}$ (respectively, $J_{j}^{i}$ ) and that $\nu_{i}$ (respectively, $\nu_{j}^{i}$ ) has exactly polynomial growth of degree $p(\mu)$.

Using the placques in $\operatorname{int}\left(B_{j}^{i}\right)$ coming from suitable biregular coordinate charts, we produce a growth function $g_{j}^{i}$ for $B_{j}^{i}$ that is simultaneously a growth function for each leaf of $\hat{\mathscr{F}}_{j}^{i}$. As in the remarks preceding (2.8), if $q_{j}^{i}-1$ is the level of the leaf on which $B_{j}^{i}$ spirals, then $g_{j}^{i}$ has exactly polynomial growth of degree $q_{j}^{i}$.

Let $\tilde{\nu}_{j}^{i}(m)$ denote the number of placques in $B_{j}^{i}(\hat{\mathcal{E}})$ that can be reached from $x$ by a connected string $x \in P_{1}, P_{2}, \ldots, P_{k}$ of placques, $k \leqslant m+1$. We need only consider strings for which there is an integer $l$ with $P_{s} \subset K, 1 \leqslant s \leqslant l-1$, $P_{l} \subset V_{j}^{i}$, and $P_{s} \subset B_{j}^{i}(\hat{\mathfrak{C}}), l+1 \leqslant s \leqslant k$. It follows that

$$
\nu_{j}^{i}(m) g_{j}^{i}(m) \leqslant \tilde{\nu}_{j}^{i}(2 m) \leqslant \nu_{j}^{i}(2 m) g_{j}^{i}(2 m) .
$$

This proves the following.

(6.12) LemMA. Each $\tilde{\nu}_{j}^{i}$ has exactly polynomial growth of degree $p(\mu)+q_{j}^{i}$.

Since the growth function for $L$ at $x$ is the sum of all $\nu_{i}$ and $\tilde{\nu}_{j}^{i},(6.10)$ follows immediately; hence (6.2) is completely proven.

REMARKS ON THE ANALYTIC CASE. Let $\mathscr{F}$ be transversely analytic. Then, if $U$ is a local minimal set of locally dense type, if $\mathscr{F} \mid U$ is without holonomy, and if $\bar{U}-U$ is totally proper, it is easy to show that $\hat{\mathscr{F}}$ is almost trivial. This is accomplished by an analysis of the holonomy along the leaves of $\bar{U}-U$, using the fact that an analytic holonomy element with infinitely many fixed points (in a compact arc) is the identity. Thus, (3.6), (3.7), and (6.10) imply that nonexponential leaves at finite level in a transversely analytic foliation must have exactly polynomial growth. But one can also show, rather easily, that, for such foliations, there is a finite upper bound to the levels of all leaves and that there are only finitely many local minimal sets of locally dense type. This results in the following.

(6.13) THEOREM. Let the (codimension one) foliation $\mathcal{F}$ of the closed manifold $M$ be transversely analytic. Then there are only finitely many growth types for the leaves of $\mathcal{F}$, the nonexponential types being exactly polynomial.

A detailed proof of (6.13) will appear elsewhere. 
7. Fractional growth. Let $s \in \mathbf{R}, s>2$, and define $\gamma_{s}: \mathbf{Z}^{+} \rightarrow \mathbf{R}^{+}$by $\gamma_{s}(m)=m^{s}$. By a suitable choice of the sequences $\{c(k)\}_{k \geqslant 1}$ and $\{N(k)\}_{k>1}$, we will construct $\mathscr{F}(h, l)$ exactly as in $\S 5$ in such a way that the open, saturated set $U$ consists of leaves having the growth type $\operatorname{gr}\left(\gamma_{s}\right)$. In this construction, $\mathscr{F}(h, l)$ will be of class $C^{1}$ and it will be $C^{1}$-trivial at the boundary. As in (5.2), one can transfer a modification of this foliation to any closed 3-manifold, the growth type of the interesting leaves being raised to $\operatorname{gr}\left(\gamma_{s+1}\right)$. This will give the following.

(7.1) THEOREM. Let $M$ be a closed 3-manifold and let $s>3$ be a real number. Then there exists a $C^{1}$-foliation $\mathcal{F}$ of $M$ having a local minimal set $U$ of locally dense type (and $\bar{U}-U$ totally proper) such that $\mathcal{F} \mid U$ has trivial holonomy and the common growth type in $\mathscr{F} \mid U$ is $\operatorname{gr}\left(\gamma_{s}\right)$.

Somewhat similar foliations are constructed in [Ts1]. In [C-C5] we show how to $C^{\infty}$-smooth the foliations in (7.1).

As in $\$ 5$, let $h:[-1,1] \rightarrow[-1,1]$ be smooth with $h(t)>t,-1<t<1$, and $C^{\infty}$-flat at \pm 1 . Fix $[\bar{a}, a] \subset(-1,1)$ such that $h(\bar{a})=a$. Let $\varphi(t) d / d t$ be a smooth vector field on $I=[-1,1]$ with $\operatorname{supp}(\varphi)=[\bar{a}, a]$ and $\varphi(t)>0$ for $\bar{a}<t<a$. Let $\Phi: \mathbf{R} \times I \rightarrow \mathbf{R} \times I$ be the flow generated by this field.

As usual, $\{c(k)\}_{k \geqslant 1}$ decreases monotonically to 0 and $\{N(k)\}_{k \geqslant 1}$ is a strictly increasing sequence of nonnegative integers. We take $N(1)=0$ and define

$$
l_{k}=h^{N(k)} \circ \Phi_{c(k)} \circ h^{-N(k)}, \quad l=\cdots \circ l_{k} \circ l_{k-1} \circ \cdots \circ l_{1} \text {. }
$$

In $\S 5$, the numbers $N(k)$ did not depend on the numbers $c(m)$, so the latter sequence could be chosen to converge to 0 so fast that $l$ was of class $C^{\infty}$. In the present construction we will not have that freedom. However, if $l$ can be shown to be smooth of some class $C^{n}, n \geqslant 1$, then it is elementary that $l \in \operatorname{Diff}_{+}^{n}(I)$ and $l$ is $C^{n}$-flat at \pm 1 .

The following proof was shown to us by G. Hector. An alternative proof is given in [C-C5, (3.2)].

(7.2) LEMMA. The group $G$ generated by $h$ and $l$ is a subgroup of $\operatorname{Diff}_{+}^{1}(I)$ and every element of $G$ is $C^{1}$-flat at \pm 1 .

PROOF. It will be sufficient to prove that the sequence $\left\{d l_{k} / d t\right\}_{k>1}$ converges uniformly to 1 on $I$. Set $I_{k}=\operatorname{supp}\left(l_{k}\right)=h^{N(k)}[\bar{a}, a]$. For $x \in I_{k}$ and $y=h^{-N(k)}(x)$ $\in[\bar{a}, a]$, we have

$$
\left(d l_{k} / d t\right)_{x}=\frac{\left(h^{N(k)}\right)^{\prime}\left(\Phi_{c(k)}(y)\right)}{\left(h^{N(k)}\right)^{\prime}(y)}\left(\Phi_{c(k)}\right)^{\prime}(y) .
$$

Since $h$ is of class $C^{\infty}$, there exists $\theta>0$ such that $\left|h^{\prime \prime}(u) / h^{\prime}(u)\right| \leqslant \theta$ uniformly on I. By a calculation that, by now, is classical (cf. [Sa, p. 83]) we obtain

$$
\log \left(\frac{\left(h^{N(k)}\right)^{\prime}\left(\Phi_{c(k)}(y)\right)}{\left(h^{N(k)}\right)^{\prime}(y)}\right) \leqslant \theta \sum_{j=0}^{N(k)-1}\left|h^{j}\left(\Phi_{c(k)}(y)\right)-h^{j}(y)\right| .
$$

But $\Phi_{c(k)}(y) \rightarrow y$ uniformly on $[\bar{a}, a]$, and the interiors of the intervals $h^{j}[\bar{a}, a]$ are disjoint, so the right-hand side of (*) tends uniformly to 0 on $[\bar{a}, a]$ as $k \rightarrow \infty$. Since $\left(\Phi_{c(k)}\right)^{\prime}(y) \rightarrow 1$ uniformly on $[\bar{a}, a]$ as $k \rightarrow \infty$, the lemma follows. 
Let $\{s(k)\}_{k \geqslant 2}$ be a sequence of integers, each $s(k) \geqslant 2$, and define $c(k)$ inductively by $c(1)=1, c(k)=c(k-1) / s(k), k \geqslant 2$.

Fix $t \in(\bar{a}, a)$. Let $G_{p} \subset \operatorname{Diff}_{+}^{\infty}(I)$ denote the group generated by $\left\{h, l_{1}, \ldots, l_{p}\right\}$ and let $g_{p}$ denote the growth function of the graph $\left|G_{p}(t)\right|$ relative to this generating set. Let $g$ denote the growth function of $|G(t)|$ relative to $\{h, l\}$. Remark that $g(m)=g_{p}(m)$ for $m \leqslant N(p+1)$.

(7.3) LEMMA. If $m \geqslant 0$ and $p \geqslant 2$, then $g_{p}(m) \leqslant s(p) g_{p-1}(m)$.

Proof. Let $\Gamma_{n}(m) \subset G_{n}(t)$ denote the subset counted by $g_{n}(m)$. We define an injection

$$
\varphi: \Gamma_{p}(m) \rightarrow\{0,1, \ldots, s(p)-1\} \times \Gamma_{p-1}(m) .
$$

The assertion will follow. If $t^{\prime} \in \Gamma_{p-1}(m) \subset \Gamma_{p}(m)$, set $\varphi\left(t^{\prime}\right)=\left(0, t^{\prime}\right)$. If $t^{\prime} \in \Gamma_{p}(m)$ $-\Gamma_{p-1}(m)$, fix an expression

$$
t^{\prime}=h^{\beta} \circ l_{p}^{\alpha(p)} \circ h^{N(p)-N(p-1)} \circ \cdots \circ l_{2}^{\alpha(2)} \circ h^{N(2)-N(1)} \circ l_{1}^{\alpha(1)}(t),
$$

such that $0 \leqslant|\alpha(q)|<s(q), 2 \leqslant q \leqslant p, \alpha(p) \neq 0$, and $|\beta|+N(p)+\sum_{i=1}^{p}|\alpha(i)|$ $\leqslant m$. Since $\alpha(p) \neq 0$, we stress that

$$
|\beta|+N(p)+\sum_{i=1}^{p-1}|\alpha(i)|<m .
$$

If $\alpha(p)>0$, set

$$
t^{*}=h^{\beta+N(p)-N(p-1)} \circ l_{p-1}^{\alpha(p-1)} \circ \cdots \circ h^{N(2)-N(1)} \circ l_{1}^{\alpha(1)}(t)
$$

an element of $\Gamma_{p-1}(m)$, and set $\varphi\left(t^{\prime}\right)=\left(\alpha(p), t^{*}\right)$. Remark that $t^{*}$ uniquely determines $\beta$ regardless of other choices. If $\alpha(p)<0$, set $\bar{\alpha}(p)=s(p)+\alpha(p) \in$ $\{1, \ldots, s(p)-1\}$ and remark that

$$
t^{\prime}=h^{\beta} \circ l_{p}^{\bar{\alpha}(p)} \circ h^{N(p)-N(p-1)} \circ l_{p-1}^{\alpha(p-1)-1} \circ \cdots \circ h^{N(2)-N(1)} \circ l_{1}^{\alpha(1)}(t)
$$

and (by (**)) that $|\beta|+|\alpha(p-1)-1|+\sum_{i=1}^{p-2}|\alpha(i)|+N(p) \leqslant m$. Set

$$
t^{*}=h^{\beta+N(p)-N(p-1)} \circ l_{p-1}^{\alpha(p-1)-1} \circ \cdots \circ h^{N(2)-N(1)} \circ l_{1}^{\alpha(1)}(t)
$$

again an element of $\Gamma_{p-1}(m)$, and set $\varphi\left(t^{\prime}\right)=\left(\bar{\alpha}(p), t^{*}\right)$. Again remark that $t^{*}$ uniquely determines $\beta$. We must show that $t^{\prime}$ can be recovered from $\varphi\left(t^{\prime}\right)=\left(q, t^{*}\right)$. Indeed, if $q=0, t^{\prime}=t^{*}$, and, if $q>0, t^{\prime}=h^{\beta} \circ l_{p}^{q} \circ h^{-\beta}\left(t^{*}\right), \beta$ being determined by $t^{*}$.

Remark that $\operatorname{gr}\left(g_{1}\right)$ is exactly polynomial of degree 2 . Thus, there are positive constants $a, b$ such that $b m^{2} \leqslant g_{1}(m) \leqslant a m^{2}, \forall m \geqslant 1$.

(7.4) Corollary. If $m \leqslant N(p+1)$, then $g(m) \leqslant(a / c(p)) m^{2}$.

PROOF. The restriction on $m$ implies that $g(m)=g_{p}(m)$. Repeated applications of (7.3) give

$$
g(m)=g_{p}(m) \leqslant s(p) g_{p-1}(m) \leqslant \cdots \leqslant(1 / c(p)) g_{1}(m)
$$

and $g_{1}(m) \leqslant a m^{2}$.

(7.5) LEMMA. Suppose that, for $p$ sufficiently large, $N(p) \geqslant s(2)+\cdots+s(p)$. For such $p$, take $m \geqslant 6 N(p)$. Then $g(m) \geqslant(b / 4 c(p)) m^{2}$. 
Proof. Since $N(1)=0, l_{1}=\Phi_{c(1)}=\Phi_{1}$. Let $k \geqslant 0$. In the interval $\left[l_{1}^{k}(t), l_{1}^{k+1}(t)\right]$ $\subset(\bar{a}, a)$, there are $1 / c(p)$ points of the form

$$
h^{-N(p)} \circ l_{p}^{\alpha(p)} \circ h^{N(p)-N(p-1)} \circ l_{p-1}^{\alpha(p-1)} \circ \cdots \circ l_{2}^{\alpha(2)} \circ h^{N(2)-N(1)}\left(l_{1}^{k}(t)\right),
$$

where $0 \leqslant \alpha(q)<s(q), 2 \leqslant q \leqslant p$. Each of these points is at a distance (in $\left|G_{p}(t)\right|$ ) of less than $2 N(p)+s(2)+\cdots+s(p)$ from $l_{1}^{k}(t)$. Thus, if $m \geqslant 3 N(p) \geqslant 2 N(p)$ $+s(2)+\cdots+s(p)$, we have

$$
g(m) \geqslant g_{p}(m) \geqslant(1 / c(p)) g_{1}(m-3 N(p)) \geqslant(b / c(p))(m-3 N(p))^{2} .
$$

Finally, if $m \geqslant 6 N(p)$, then $m-3 N(p) \geqslant m / 2$, so $g(m) \geqslant(b / c(p))(m / 2)^{2}$.

Now we specify the data $s(p), N(p)$. We take $s(p)=2$, all $p \geqslant 2$; hence $1 / c(p)=2^{p-1}$. For a fixed choice of the real number $r>0$, there exists an integer $p_{0}>0$ such that $2^{r p_{0}}>p_{0}$, and, if $p>p_{0},\left[2^{r p}\right]>\left[2^{r(p-1)}\right]$. We define

$$
\begin{aligned}
& N(1)=0, \\
& N(p)=p, \quad 2 \leqslant p \leqslant p_{0}, \\
& N(p)=\left[2^{r p}\right], \quad p>p_{0} .
\end{aligned}
$$

For $p$ large enough, $N(p) \geqslant 2(p-1)=s(2)+\cdots+s(p)$, so (7.5) applies with this choice of data.

(7.6) LEMMA. With the above choices, $(a / 2) m^{2+(1 / r)}>g(m)>b_{*} m^{2+(1 / r)}$, for $a$ suitable positive constant $b_{*}$ and all sufficiently large values of $m$.

Proof. For each $m \geqslant 1$, there is a unique value of $p$ for which $N(p)<m \leqslant$ $N(p+1)$. By choosing $m$ large enough, one forces $p$ to be as large as desired. In particular, we can assume $N(p)=\left[2^{r p}\right]$, so $m>2^{r p}$; hence $m^{1 / r}>2^{p}$. By (7.4),

$$
g(m) \leqslant(a / c(p)) m^{2}=a 2^{p-1} m^{2}<(a / 2) m^{2+(1 / r)} .
$$

For large $m$, there is a unique value of $p$ such that $6 N(p)<m \leqslant 6 N(p+1)$, again forcing $p$ to be large. By $(7.5), g(m) \geqslant(b / 4 c(p)) m^{2}=(b / 16) 2^{p+1} m^{2}$. But $m \leqslant$ $6 N(p+1) \leqslant 6 \cdot 2^{r(p+1)}$, so $m^{1 / r} / 6^{1 / r} \leqslant 2^{p+1}$. Thus, take $b_{*}=(b / 16) 6^{-1 / r}$ and obtain $g(m) \geqslant b_{*} m^{2+(1 / r)}$.

As $r$ ranges over $(0, \infty), s=2+(1 / r)$ ranges over $(2, \infty)$. Thus, for each such $s$ we have constructed a $C^{1}$-foliation $\mathscr{F}(h, l)$ such that the leaves in $U$ have the growth type of $\gamma_{s}$. This completes the proof of (7.1).

\section{REFERENCES}

[A-S] L. V. Ahlfors and L. Sario, Riemann surfaces, Chapter I, 86, Princeton Univ. Press, Princeton, N. J., 1960.

[C] L. Conlon, Transversally parallelizable foliations of codimension two, Trans. Amer. Math. Soc. 194 (1974), 79-102.

[C-C1] J. Cantwell and L. Conlon, Leaf prescriptions for closed 3-manifolds, Trans. Amer. Math. Soc. 236 (1978), 239-261.

[C-C2] _ Leaves with isolated ends in foliated 3-manifolds, Topology 16 (1977), 311-322.

[C-C3] _ Growth of leaves, Comment. Math. Helv. 53 (1978), 93-111.

[C-C4] _ Poincaré-Bendixson theory for leaves of codimension one, Trans. Amer. Math. Soc. 265 (1981), 181-209.

[C-C5] __ Smoothing fractional growth, Tôhoku Math. J. 33 (1981), 249-262.

[D] P. Dippolito, Codimension one foliations of closed manifolds, Ann. of Math. (2) 107 (1978), 403-453. 
[F] H. Freudenthal, Über die Enden topologischer Räume und Gruppen, Math. Z. 33 (1931), 692-713.

[G] S. Goodman, Closed leaves in foliations of codimension one, Comment. Math. Helv. 50 (1975), 383-388.

[He1] G. Hector, Leaves whose growth is neither exponential nor polynomial, Topology 16 (1977), 451-459.

[He2] _ Croissance des feuilletages presque sans holonomie, Differential Topology, Foliations, and Gelfand-Fuks Cohomology, Proc. Sympos. (Rio de Janeiro, 1976), Lecture Notes in Math., vol. 652, Springer-Verlag, New York, 1978.

[Ho] H. Hopf, Enden offener Räume und unendliche diskontinuierliche Gruppen, Comment. Math. Helv. 16 (1944), 81-100.

[L] C. Lamoureux, Sur quelques phénomènes de captage, Ann. Inst. Fourier (Grenoble) 23 (4) (1973), 229-243.

[P] J. Plante, Foliations with measure preserving holonomy, Ann. of Math. (2) 102 (1975), 327-361.

[Po] L. Pontryagin, Topological groups, 2nd ed., Gordon and Breach, New York, 1966.

[R] W. Rudin, Principles of mathematical analysis, 3rd ed., Internat. Ser. Pure and Appl. Math., McGraw-Hill, New York, 1976.

[Sa] R. Sacksteder, Foliations and pseudogroups, Amer. J. Math. 87 (1965), 79-102.

[Sc] P. Schweitzer, Counterexamples to the Seifert conjecture and opening closed leaves of foliations, Ann. of Math. (2) 100 (1974), 386-400.

[Th] W. Thurston, A local construction of foliations for three-manifolds, Proc. Sympos. Pure Math., vol. 27, Amer. Math. Soc., Providence, R. I., 1975, pp. 315-319.

[TI] D. Tischler, On fibering certain foliated manifolds over $S^{1}$, Topology 9 (1970), 153-154.

[Ts1] N. Tsuchiya, Leaves with non-exact polynomial growth, Tôhoku Math. J. 32 (1980), 71-77.

[Ts2] __, Growth and depth of leaves, J. Fac. Sci. Univ. Tokyo Sect. IA Math. 26 (1979), 473-500.

Department of Mathematics, St. Louis University, St. Louis, Missouri 63103

Department of Mathematics, Washington University, St. Louis, Missouri 63130 